\title{
Risk of Depression and Suicidality among Diabetic Patients: A Systematic Review and Meta-Analysis
}

\author{
Rasha Elamoshy ${ }^{1}$, Yelena Bird ${ }^{1}$, Lilian Thorpe ${ }^{2}$ and John Moraros ${ }^{1, *}$ \\ 1 School of Public Health, University of Saskatchewan, Saskatoon, SK S7N 2Z4, Canada; \\ rasha.elamoshy@usask.ca (R.E.); yelena.bird@usask.ca (Y.B.) \\ 2 Community Health and Epidemiology, University of Saskatchewan, Saskatoon, SK S7N 2Z4, Canada; \\ lilian.thorpe@usask.ca \\ * Correspondence: john.moraros@usask.ca; Tel.: +1-306-966-8578
}

Received: 22 October 2018; Accepted: 14 November 2018; Published: 16 November 2018

check for updates

\begin{abstract}
The purpose of this study is to conduct a systematic review and meta-analysis to evaluate the risk of depression and suicidality among diabetic patients. Methods: Medline, PubMed, EMBASE, Cochrane library, and Psych INFO were searched for studies published from 2008 onwards. Meta-analysis was conducted to estimate the pooled effect size. Sources of heterogeneity were investigated by subgroup analysis and meta-regression. Results: In total, 5750 articles were identified and of those, 17 studies on suicidality and 36 on depression were included in this study. Our analysis suggests a positive relationship between diabetes and depression (cohort studies odds ratio (OR) $1.49,95 \%$ confidence interval (CI): 1.36-1.64 and cross-sectional studies OR 2.04, 95\% CI, 1.73-2.42). Pooled OR values for suicidal ideation, attempted suicide, and completed suicide were 1.89 (95\% CI: 1.36-2.63), 1.45 (95\% CI: 1.07-1.96), and 1.85 (95\% CI: 0.97-3.52), respectively. All findings were statistically significant except for completed suicide. Conclusions: The increased risk of depression and suicidality in diabetic patients highlights the importance of integrating the evaluation and treatment of depression with diabetes management in primary healthcare settings. Further research in this area is needed.
\end{abstract}

Keywords: diabetes; depression; suicide; suicidal ideation; suicidal attempts; suicidal death

\section{Introduction}

Diabetes is considered one of the largest global epidemics and constitutes a public health emergency in many countries [1]. The global prevalence of diabetes among adults has nearly doubled in the last couple of decades, rising from $4.7 \%$ in 1980 to $8.5 \%$ in 2014, resulting in approximately 1.6 million deaths annually [2]. Diabetes is psychologically demanding given the chronic and large burden placed on diabetics for the self-management of their disease. Diabetic patients face several psychological challenges as a result of their illness, which may include: adherence to medical treatment and lifestyle modifications, need for continued monitoring for glycemic control, concerns for complications and disabilities, interference of symptoms with daily activities, and psychosocial difficulties at personal and interpersonal levels [3], all of which may eventually lead and interconnect to depression and in some cases suicide.

Depression is a common mental illness that negatively impacts productivity and quality of life [4]. It is reported that patients with depression die 5-10 years earlier than those without depression [5]. Evidence suggests that the co-morbidity of depression and diabetes is relatively common [6-8]. A previous meta-analysis estimated the prevalence of depression to be twice as high among diabetics compared to the general population [6]. Other systematic reviews corroborated these findings and 
demonstrated a significantly higher risk for diabetic patients to develop depression compared to non-diabetics $[7,8]$.

A recent study found no genetic link to account for the positive association between diabetes and depression [9]. However, certain epigenetic factors (low socioeconomic status, irregular sleep patterns, lack of physical activity, and poor diet) may play an important role in activating common physiological pathways (stress and inflammation systems) that promote and reinforce diabetes and depression [10]. Chronic stress activates the hypothalamus-pituitary-adrenal axis (HPA axis) and the sympathetic nervous system (SNS), leading to increased production of cortisol and adrenalin/noradrenalin, respectively [11]. Chronic levels of increased cortisol and prolonged secretion of adrenalin/noradrenalin may promote diabetes (insulin resistance) [12] and depression (dopamine dysfunction) [13] or both (disturbance of neurogenesis in the hippocampus) [14]. Furthermore, chronic stress increases the production of pro-inflammatory cytokines that can lead to diabetes (by disturbing the normal functioning of the pancreatic $\beta$-cells) [15] and depression (by disrupting neuroendocrine function) [16].

The co-morbidity between diabetes and depression is associated with poorer prognosis and higher medical expenditures than either condition alone [17]. This is an expected finding as depression significantly impacts diabetic patients leading to poor self-care, non-adherence to medical treatment, reduced quality of life, higher rates of morbidity and mortality, and consequently, increased healthcare costs $[18,19]$. Additionally, depression among diabetic patients tends to last longer and has higher recurrence rates compared to non-diabetics [20]. Consequently, identification of depression among diabetic patients is critically important to mitigate these negative personal consequences and realize cost-savings in healthcare.

Suicide was responsible for more than 800,000 deaths per year in 2015 and is the second leading cause of death among those aged 15 to 29 years old worldwide [21]. Depressive symptoms may increase the risk of diabetic patients displaying suicidality [22], which may include: suicidal ideation, attempted suicide, and completed suicide. Although the association between diabetes and depression has been previously examined, the association with suicidality remains unclear and the evidence is limited and at times, conflicting [23-25].

Despite the documented interconnections, there is a significant gap in the literature regarding the systematic assessment of depression and suicidality in patients with diabetes. To the best of our knowledge, the present study is one of the first meta-analyses of its kind and adds value to our understanding on this important research topic. The purpose of this study was to conduct a systematic synthesis and meta-analysis of the evidence in order to: (1) assess the risk of depression (including clinical depression, depressive symptoms, and use of antidepressants); (2) determine the prevalence of suicidality; and (3) evaluate the risk of suicidality among diabetic patients.

\section{Experimental Section}

\subsection{Data Sources and Search Strategies}

A systematic literature search was undertaken using five relevant databases: PubMed, Medline, EMBASE, Psych INFO, and Cochrane Library. Search strategies focused on three major domains: (1) diabetes; (2) depression or suicidality; and (3) quantitative outcome measures.

\subsection{Eligibility Criteria and Study Selection}

In our study, eligible articles were required to: (1) be published in the English language in peer-reviewed journals since 2008, and be available in full text; (2) assess patients with type 1 and/or type 2 diabetes mellitus who either self-reported a physician's diagnosis of diabetes, were prescribed anti-diabetic medications, or were participants in studies using laboratory-based assessments; and (3) evaluate depressive disorders, or use of antidepressants or depressive symptoms based on validated standardized questionnaires. Studies were also selected by scanning the reference 
lists of previous systematic reviews on the topic. Articles had to include both exposed and non-exposed groups of participants and provide sufficient data to calculate odds ratio for depression among diabetics. Eligible articles were identified through title and abstract screening, followed by full text review. Two reviewers independently evaluated studies for relevance in a standardized manner. Non-agreement was resolved by discussion and adjudication.

\subsection{Data Extraction}

Data extraction strategies were developed and pilot-tested on 20 randomly selected included studies and then modified accordingly. Information extracted in duplicate from studies included: author, publication year, country, study design, follow-up time (for cohort studies), settings, total number, age and sex of study participants, method of diabetes evaluation, method of depression or suicidality assessment, reported effect measure with $95 \%$ confidence interval, and covariates for adjusted effect measures. Unadjusted odds ratios and $95 \%$ confidence intervals were calculated using the number of events in the exposed and non-exposed groups.

\subsection{Risk of Bias}

Two reviewers independently assessed the validity of eligible studies by using a modified version of the Newcastle Ottawa Scale (NOS) [26]. The NOS assesses representativeness of the study sample, comparability between respondents and non-respondents, ascertainment of depression or suicidality, and thoroughness of the reported descriptive statistics. Studies were rated as having a low, moderate, or high risk of bias.

\subsection{Data Analysis}

Statistical analysis was performed using the Comprehensive Meta-analysis software-version 3 (CMA-3) [27]. For depression, pooled odds ratios (both adjusted and unadjusted) were the effect measure of interest. While for suicidality, both odds ratios and pooled prevalence among diabetics were the focus of our analysis. As heterogeneity was likely to exist, a random effect model was used to calculate pooled estimates, which allows for estimating both within and between studies variation. We examined heterogeneity using the Cochran's $Q$ heterogeneity test and $\mathrm{I}^{2}$ as a measure for inconsistency [28]. Influential analysis was conducted for the effect of each study on the pooled estimate by reassessing estimates after removal of one study at the time. To visually assess for publication bias, the funnel plot method was used. The Egger's regression intercept method was also used to confirm and statistically test for the bias observed in the funnel plot [29]. When publication bias was detected, a Duval and Tweedie trim and fill method was used to calculate the adjusted effect size [30]. All analysis used a $5 \%$ level of significance $(\alpha=0.05)$.

\section{Results}

\subsection{Study Selection}

The PRISMA flow chart depicting the study selection is shown in Figure 1. A comprehensive search of the literature yielded a total of 5750 articles from which 427 articles qualified for full text screening and 355 were excluded due to one or more of the following conditions: (1) their full text was not available; (2) they were not written in English; (3) they focused on special populations; and/or (4) reported inadequate data or mixed outcomes. In total, 50 studies were analyzed, with 33 reporting data only on depression, 14 only on suicidality, and three studies reporting data on both depression and suicidality among diabetic patients [31-33]. 


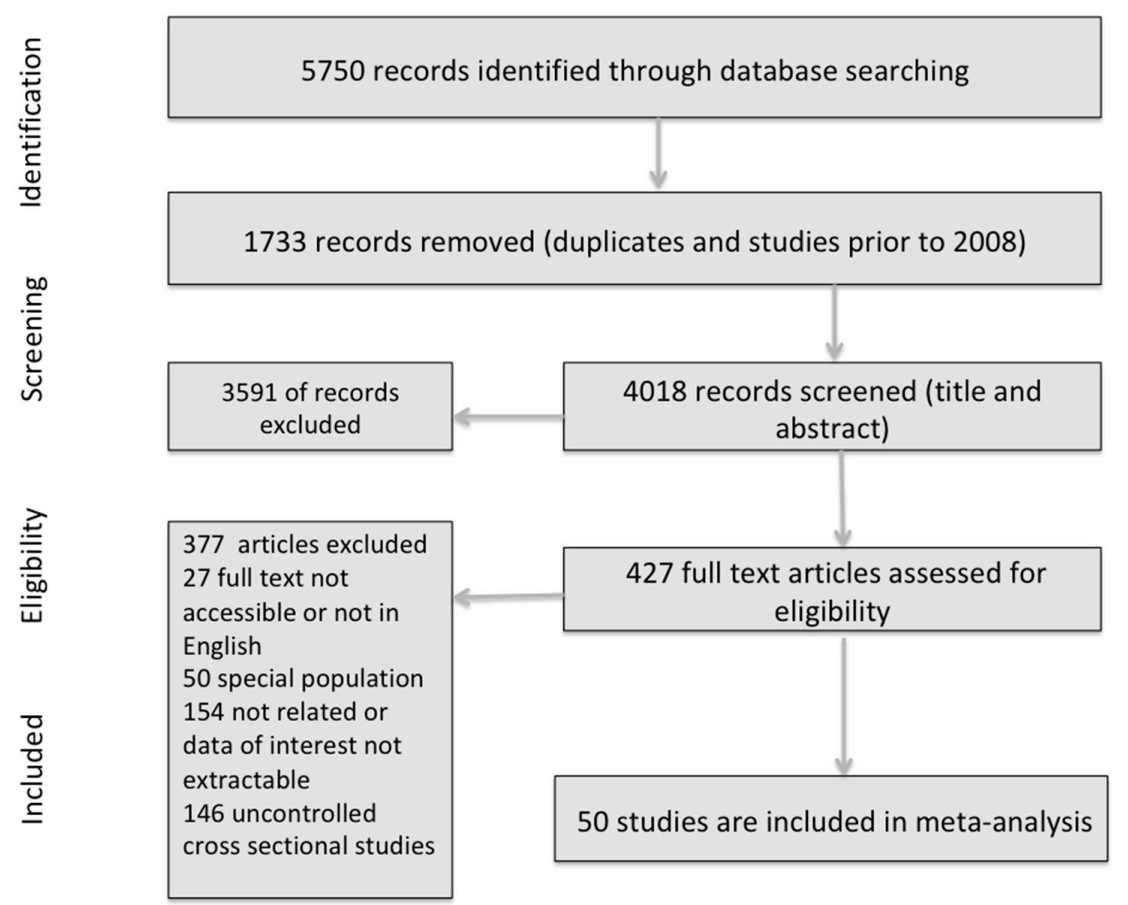

Figure 1. Preferred Reporting Items for Systematic Reviews and Meta-Analyses (PRISMA) flow chart for study selection process.

\subsection{Study Characteristics}

\subsubsection{Depression}

Depression studies were stratified and examined on the bases of their study design. Tables 1 and 2 include summary of the characteristics of depression studies included in the review.

\section{(1) Cohort Studies}

There were 12 cohort studies included for depression. One study [34] reported two datasets for patients with prevalent and incident diabetes, leaving a total of 13 datasets for analysis. Three studies [35-37] used incident prescription of antidepressants as a proxy for depression diagnosis, five studies [34,38-41] used clinical diagnostic criteria, and three [42-44] relied on questionnaires. One study used both questionnaires and prescription of antidepressants [42]. The follow-up period ranged from two [34] to 15 years [36]. Most studies were from Europe or North America, while only two studies were from Asia $[38,39]$. Ascertainment of diabetes was made by physician diagnosis (International Classification of Diseases (ICD) code) [34,36,38-40,43], use of anti-diabetic medications [35,37,43], self-reported diagnosis of diabetes [44,45], and/or laboratory assessment [42,43].

\section{(2) Cross-Sectional Studies}

There were 23 cross-sectional studies and one case-control study [46] on depression and diabetes. Additionally, three of these studies reported data on suicidality [31-33]. Depression was evaluated by: (1) questionnaires; (2) clinical diagnostic criteria (nine studies) [31,32,46-52]; and (3) utilization of antidepressants (one study) [53]. Two studies [47,54] used both depressive disorders and symptoms but for the analysis, we used only results for depressive disorders.

\subsubsection{Suicidality}

Suicidality was stratified and examined on the bases of outcome. Tables 3 and 4 include summary of the characteristics of suicidality studies included in the review. 
Table 1. Characteristics of selected depression studies.

\begin{tabular}{|c|c|c|c|c|c|c|c|c|c|}
\hline Author \&Year & Country & Study Design & Setting & Patients $N$ & Age & Female \% & Type of Diabetes & $\begin{array}{l}\text { Outcome } \\
\text { Reported }\end{array}$ & $\begin{array}{l}\text { Evaluation of } \\
\text { Outcome }\end{array}$ \\
\hline Icks et al., 2013 [43] & Germany & $\begin{array}{l}\text { Prospective } \\
\text { cohort }\end{array}$ & $\begin{array}{l}\text { Population based, mandatory } \\
\text { residence in some cities }\end{array}$ & 3663 & $45-75$ & 48.6 & $\begin{array}{l}\text { Not specified, (included } \\
\text { diagnosed, undiagnosed, and } \\
\text { impaired glucose } \\
\text { tolerance [IGT]) }\end{array}$ & Depression & $\begin{array}{c}\text { Center for } \\
\text { Epidemiologic } \\
\text { Studies-Depression } \\
\text { Scale (CES-D) }(15 \\
\text { items) } \geq 17\end{array}$ \\
\hline Cleal et al., 2017 [36] & Netherland & $\begin{array}{c}\text { Prospective } \\
\text { cohort }\end{array}$ & Population registers & $3,434,420$ & $18-59$ & 48.9 & Not specified Incident diabetes & Depression & $\begin{array}{l}\text { Prescription of } \\
\text { antidepressants }\end{array}$ \\
\hline $\begin{array}{l}\mathrm{O}^{\prime} \text { Connor et al., } \\
2009[34]\end{array}$ & USA & $\begin{array}{l}\text { Historical cohort } \\
2 \text { years }\end{array}$ & $\begin{array}{l}\text { Patients enrolled in a health plan, } \\
\text { services provided by primary } \\
\text { care, family physicians and } \\
\text { general internists }\end{array}$ & $\begin{array}{c}2932 \text { with incident } \\
\text { diabetes and } 14,144 \\
\text { with prevalent diabetes } \\
\text { with equal number of } \\
\text { matched controls }\end{array}$ & $\begin{array}{l}\geq 40 \text { Mean around } \\
61\end{array}$ & $47.3 \%$ & $\begin{array}{l}\text { Not specified but majority were } \\
\text { type } 2 \text { International } \\
\text { Classification of Diseases, ninth } \\
\text { version (ICD-9) }\end{array}$ & Depression & $\begin{array}{c}\text { ICD-9 } \pm \\
\text { antidepressants }\end{array}$ \\
\hline Chen et al., 2013 [38] & Taiwan & Cohort & $\begin{array}{l}\text { National health insurance claims } \\
\text { and data linkage }\end{array}$ & & Mean $60.1 \pm 13.2$ & 46.5 & $\begin{array}{l}\text { Type } 2 \text { ICD-9 Clinical } \\
\text { Modification (CM) }\end{array}$ & Depression & ICD-9 CM \\
\hline Hamer et al., 2011 [45] & UK & $\begin{array}{l}\text { Prospective } \\
\text { cohort }\end{array}$ & $\begin{array}{c}\text { Community dwelling with older } \\
\text { adults }\end{array}$ & 4338 & Mean $62.9 \pm 9$ & $45.2 \%$ & $\begin{array}{l}\text { Not specified Self report } \\
\text { physician diagnosis }\end{array}$ & Depression & $\begin{array}{l}\text { CES-D ( } 8 \text { items) With a } \\
\text { cut off } \geq 4\end{array}$ \\
\hline Hsu et al., 2011 [40] & Taiwan & $\begin{array}{l}\text { Cohort Median } \\
\text { follow-up } 6.5 \\
\text { years. }\end{array}$ & $\begin{array}{l}\text { Claim data, national health } \\
\text { insurance program }\end{array}$ & $\begin{array}{l}\text { 14,048 diabetics and } \\
55,608 \text { control }\end{array}$ & $\geq 20$ & & $\begin{array}{l}\text { Not specified (incident diabetes) } \\
\text { ICD-9 CM }\end{array}$ & Depression & ICD-9 CM \\
\hline Golden et al., 2008 [42] & USA & $\begin{array}{c}\text { Prospective } \\
\text { cohort } 3.1 \text { years. }\end{array}$ & $\begin{array}{l}\text { Part of multi ethnic study of } \\
\text { atherosclerosis }\end{array}$ & 4847 & $45-84$ & & $\begin{array}{l}\text { Type 2, fasting blood glucose } \\
\text { (FBG) } \geq 126 \text { or on oral } \\
\text { hypoglycemic agent (OHA) or } \\
\text { Insulin }\end{array}$ & Depression & $\begin{array}{l}\text { CED-D } \geq 16 \text { or use of } \\
\text { antidepressant } \\
\text { medications or both }\end{array}$ \\
\hline Huang et al., 2012 [39] & Taiwan & $\begin{array}{l}\text { Prospective } \\
\text { cohort 4 years. }\end{array}$ & Service claim records & 200,432 & & & Not specified ICD-9 CM & Depression & ICD-9 CM \\
\hline $\begin{array}{l}\text { Demakakos et al., } \\
2014[44]\end{array}$ & UK & $\begin{array}{l}\text { Prospective } \\
\text { cohort }\end{array}$ & Community dwellings & 4238 & $\geq 50$ & & $\begin{array}{l}\text { Not specified Self report } \\
\text { physician diagnosis }\end{array}$ & Depression & $\begin{array}{l}\text { CES-D ( } 8 \text { items) With a } \\
\text { cut off } \geq 4\end{array}$ \\
\hline Knol et al., 2009 [37] & Netherland & Cohort & Pharmacy registry database & $\begin{array}{l}\text { 49,593 diabetics and } \\
154,441 \text { non diabetics }\end{array}$ & $>40$ & & Not specified Incident diabetes & Depression & $\begin{array}{l}\text { Incident use of } \\
\text { antidepressants }\end{array}$ \\
\hline Aarts et al., 2010 [41] & Netherland & $\begin{array}{l}\text { Retrospective } \\
\text { cohort } 7.7-7.9 \\
\text { years. }\end{array}$ & General practice patients & $\begin{array}{l}6140 \text { diabetics and } \\
18,416 \text { control }\end{array}$ & $\begin{array}{c}>40-97 \\
\text { Mean } 63.8 \pm 11.2\end{array}$ & $\begin{array}{c}51 \% \text { among cases } \\
\text { and } 53 \% \text { among } \\
\text { control }\end{array}$ & $\begin{array}{l}\text { Type } 2 \text { International } \\
\text { Classification of Primary Care } \\
\text { (ICPC) diagnosis based on FBG } \\
>124\end{array}$ & Depression & $\begin{array}{l}\text { ICPC code through } \\
\text { diagnostic interview }\end{array}$ \\
\hline $\begin{array}{l}\text { Kivimaki et al., } \\
\quad 2010[35]\end{array}$ & Finland & Cohort & Employees Record linkage & $\begin{array}{l}493 \text { diabetics and } 2450 \\
\text { control }\end{array}$ & $25-65$ & $58 \%$ & $\begin{array}{l}\text { Type } 2 \text { Incident diabetes, first } \\
\text { diagnosed as eligible to } \\
\text { treatment }\end{array}$ & Depression & Antidepressants \\
\hline Ryu et al., 2016 [46] & USA & $\begin{array}{c}\text { Nested } \\
\text { case-control }\end{array}$ & $\begin{array}{l}\text { Electronic health records of } \\
\text { primary care patients }\end{array}$ & $\begin{array}{l}\text { Cases with Major } \\
\text { Depressive Disorders } \\
\text { (MDD) }=11,375 \text { and } \\
\text { equal number of } \\
\text { controls }\end{array}$ & Median age 43 & $65 \%$ & $\begin{array}{l}\text { Not specified At least two } \\
\text { diagnostic codes for the } \\
\text { condition }>30 \text { days apart }\end{array}$ & Depression & $\begin{array}{c}\geq 2 \text { MDD-related } \\
\text { (ICD-9-CM) diagnosis } \\
\text { codes, } \geq 1 \\
\text { anti-depressant } \\
\text { prescription, } \geq 1 \\
\text { mention of MDD } \\
\text { diagnoses within } \\
\text { inpatient or outpatient }\end{array}$ \\
\hline
\end{tabular}


Table 1. Cont.

\begin{tabular}{|c|c|c|c|c|c|c|c|c|c|}
\hline Author \&Year & Country & Study Design & Setting & Patients $N$ & Age & Female \% & Type of Diabetes & $\begin{array}{l}\text { Outcome } \\
\text { Reported }\end{array}$ & Evaluation of Outcome \\
\hline Icks et al., 2008 [55] & Germany & Cross-sectional & $\begin{array}{l}\text { Baseline data from German } \\
\text { Heinz Nixdorf Recall study }\end{array}$ & $\begin{array}{l}2090 \text { diabetic and } 4595 \\
\text { non diabetic }\end{array}$ & $45-75$ & $50.2 \%$ & $\begin{array}{l}\text { Not specified Self report } \\
\text { physician diagnosis or } \\
\text { medications, or FBG and } \\
\text { random blood glucose (RBG) }\end{array}$ & Depression & CES-D short form $\geq 15$ \\
\hline James et al., $2010[47]$ & Nigeria & Cross-sectional & $\begin{array}{l}\text { Outpatient clinic in tertiary } \\
\text { center }\end{array}$ & $\begin{array}{l}200 \text { cases and } 200 \\
\text { control }\end{array}$ & $\begin{array}{c}20-64 \text { Mean } 47.1 \\
\quad \pm 9.6\end{array}$ & $54 \%$ & $\begin{array}{l}\text { Not specified, diagnosed for }>1 \\
\text { year Based on WHO criteria }\end{array}$ & Depression & $\begin{array}{l}\text { Schedule for the Clinical } \\
\text { Assessment in } \\
\text { Neuropsychiatry (SCAN) } \\
\text { and Beck Depression } \\
\text { Inventory (BDI) (21 } \\
\text { items) } \geq 10\end{array}$ \\
\hline Bruce et al., 2016 [56] & Australia & Cross-sectional & $\begin{array}{l}\text { Prior involvement in } \\
\text { Brusselton Health Survey } \\
\text { (community based study) }\end{array}$ & $\begin{array}{l}184 \text { cases and } 184 \\
\text { paired controls }\end{array}$ & Mean $70.2 \pm 10.1$ & $50 \%$ & Type 2 Self report and FBG & Depression & $\begin{array}{c}\text { Patient Health Questionnaire } \\
\text { (PHQ-9) and Brief Lifetime } \\
\text { Depression Scale (BLDS) } \\
\text { according to Diagnostic and } \\
\text { Statistical Manual for Mental } \\
\text { Disorders (DSM-VI) criteria } \\
\text { for major and minor } \\
\text { depression }\end{array}$ \\
\hline Lin et al., 2008 [48] & 17 countries & Cross-sectional & Household residing adults & 42,697 & $\begin{array}{l}\text { Mean between } \\
35.8-48.2\end{array}$ & $\begin{array}{c}\text { Between } \\
47.5 \%-55.1 \%\end{array}$ & $\begin{array}{l}\text { Not specified Self report } \\
\text { physician diagnosis or } \\
\text { medications }\end{array}$ & Depression & $\begin{array}{l}\text { Composite International } \\
\text { Diagnostic Interview (CIDI) }\end{array}$ \\
\hline $\begin{array}{l}\text { Van Doreen et al., } \\
2016[54]\end{array}$ & Netherland & Cross-sectional & $\begin{array}{l}\text { Baseline for a population } \\
\text { based study }\end{array}$ & 862 & Mean $64 \pm 7$ & $\begin{array}{l}30 \% \text { in diabetics } \\
\text { and } 51 \% \text { in non- } \\
\text { diabetics }\end{array}$ & $\begin{array}{c}\text { Type } 2 \text { on Insulin or Oral } \\
\text { Glucose Tolerance Test (OGTT) }\end{array}$ & Depression & $\begin{array}{c}\text { Mini-International } \\
\text { Neuropsychiatric Interview } \\
\text { (MINI) and PHQ-9 } \geq 10\end{array}$ \\
\hline Foran et al., 2015 [57] & Ireland & Cross-sectional & $\begin{array}{c}\text { Part of Cardiovascular } \\
\text { Multi-morbidity in Primary } \\
\text { Care study }\end{array}$ & $\begin{array}{l}283 \text { diabetic and } 283 \\
\text { non diabetic }\end{array}$ & $\begin{array}{c}>50 \\
\text { Mean } 68 \pm 9.5\end{array}$ & $41 \%$ & Type 2 & Depression & $\begin{array}{l}\text { Hospital Anxiety Depression } \\
\text { Scale-Depression (HADS-D) }\end{array}$ \\
\hline Chung et al., 2014 [31] & Korea & Cross-sectional & $\begin{array}{l}\text { Korean National Health and } \\
\text { Nutrition Examination } \\
\text { Survey (KNHANES IV, V) }\end{array}$ & 34,056 & $\geq 20$ & $57.1 \%$ & $\begin{array}{c}\text { Not specified Self report } \\
\text { diagnosis, FBG } \geq 126 \text {, current } \\
\text { use of anti-diabetic } \\
\text { medications }\end{array}$ & Depression & $\begin{array}{l}\text { Composite International } \\
\text { Diagnostic Interview-Short } \\
\text { Form (CIDI-SF) }\end{array}$ \\
\hline $\begin{array}{l}\text { Albertorio-Diaz et al., } \\
2017 \text { [58] }\end{array}$ & USA & Cross-sectional & NHANES data 2007-2012 & 7717 & $\geq 20$ & & $\begin{array}{c}\text { Type } 1 \& 2 \text { Self report } \\
\text { diagnosis and lab evaluation }\end{array}$ & Depression & $\begin{array}{l}\text { PHQ-9, DSM-IV text revision } \\
\text { (TR) diagnostic criteria }\end{array}$ \\
\hline Berg et al., 2012 [53] & Norway & Cross-sectional & $\begin{array}{l}\text { Norwegian prescription } \\
\text { database }\end{array}$ & $34,342,333$ & $\geq 20$ & $50.9 \%$ & $\begin{array}{l}\text { Not specified On anti-diabetic } \\
\text { treatment }\end{array}$ & Depression & Antidepressants \\
\hline Meurs et al., 2016 [49] & Netherland & Cross-sectional & $\begin{array}{l}\text { Lifeline cohort study } \\
\text { population }\end{array}$ & 90,686 & 18-93 Mean 45 & $59 \%$ & $\begin{array}{c}\text { Not specified Self-reported use } \\
\text { of anti-diabetic medication or } \\
\text { diagnosis of diabetes }\end{array}$ & Depression & MINI \\
\hline $\begin{array}{l}\text { Mantyselka et al., } 2011 \\
\text { [59] }\end{array}$ & Finland & Cross-sectional & $\begin{array}{l}\text { Based on population survey, } \\
\text { subjects }\end{array}$ & 2712 & $45-74$ & & Type 2 Self report diagnosis & Depression & $\mathrm{BDI} \geq 10$ and $\geq 16$ \\
\hline Clarke et al., 2016 [50] & UK & Cross-sectional & Scottish family health study & 23,690 & $>18$ & $51.2 \%$ & $\begin{array}{l}\text { Type } 2 \text { Self report diagnosis } \\
\text { and medication use }\end{array}$ & Depression & $\begin{array}{l}\text { Structured Clinical Interview } \\
\text { for DSM (SCID) }\end{array}$ \\
\hline $\begin{array}{l}\text { Bouwman et al., } \\
2010[60]\end{array}$ & Netherland & Cross-sectional & & 2667 & $40-65$ & $46.4 \%$ & $\begin{array}{c}\text { Type } 2 \text { FBG }>7 \mathrm{mmol} / \mathrm{L} \text { or } \\
2 \mathrm{hrPG} 11.1 \mathrm{mmol} / \mathrm{L}\end{array}$ & Depression & CES-D $\geq 16$ \\
\hline
\end{tabular}


Table 1. Cont

\begin{tabular}{|c|c|c|c|c|c|c|c|c|c|}
\hline Author \&Year & Country & Study Design & Setting & Patients $N$ & Age & Female \% & Type of Diabetes & $\begin{array}{l}\text { Outcome } \\
\text { Reported }\end{array}$ & Evaluation of Outcome \\
\hline Li et al., 2016 [61] & China & Cross-sectional & & 11,531 & $\geq 35$ & & $\begin{array}{l}\text { Not specified fasting plasma glucose } \\
(\mathrm{FPG}) \geq 7 \mathrm{mmol} / \mathrm{L} \text { or previous } \\
\text { diagnosis by a medical practitioner }\end{array}$ & Depression & PHQ-9 $\geq 10$ \\
\hline Saglam et al., 2010 [62] & Turkey & Cross- sectional & Outpatient diabetes clinic & $\begin{array}{l}500 \text { diabetic patients } \\
\text { and } 90 \text { control }\end{array}$ & $35-65$ & & $\begin{array}{c}\text { Type } 1 \text { \& } 2 \text { Known diabetics for at } \\
\text { least } 1 \text { yr. }\end{array}$ & Depression & BDI (21 items) >13 \\
\hline Kim et al., 2015 [63] & USA & Cross-sectional & $\begin{array}{l}\text { NHANES 2007-2008 and } \\
2009-2010\end{array}$ & 2266 & $20-79$ & & Not specified Self report diagnosis & Depression & PHQ-9 $\geq 10$ \\
\hline Islam et al., 2015 [64] & Bangladesh & Cross-sectional & Tertiary hospital attendants & $\begin{array}{l}591 \text { cases and } 591 \\
\text { control }\end{array}$ & $\begin{array}{c}20-60 \\
\text { Mean } 50.4 \pm 11.4\end{array}$ & $57 \%$ & $\begin{array}{l}\text { Not specified Attending physician } \\
\text { diagnosis }\end{array}$ & Depression & PHQ-9 $\geq 10$ \\
\hline Wiltink et al., 2014 [65] & Germany & Cross-sectional & $\begin{array}{l}\text { Gutenberg health study } \\
\text { population }\end{array}$ & 15,010 & 35-74 Mean 55 & $50.4 \%$ & $\begin{array}{c}\text { Not specified Self-reported } \\
\text { diagnosis and FBG >126 or RBG } \\
>200\end{array}$ & Depression & PHQ-9 $\geq 10$ \\
\hline Bessel et al., 2016 [51] & Brazil & Cross-sectional & $\begin{array}{l}\text { Civil servants active or } \\
\text { retired }\end{array}$ & 14,447 & $35-74$ & $54.1 \%$ & $\begin{array}{l}\text { Not specified Self report diagnosis, } \\
\text { medication use, HbA1c, OGTT }\end{array}$ & Depression & $\begin{array}{c}\text { Clinical Interview } \\
\text { Schedule-Revised (CIS-R) } \\
\text { clinical interview criteria } \\
\text { revised } \\
\end{array}$ \\
\hline $\begin{array}{l}\text { Adriaanse et al., } \\
2008[66]\end{array}$ & Netherland & Cross-sectional & The Hoorn study population & 550 & $69.5 \pm 6.3$ & $49.8 \%$ & Type 2, OGTT or on treatment & Depression & CES-D $\geq 16$ \\
\hline Westra et al., 2016 [67] & Netherland & Cross-sectional & & 527 & $60-87$ & & $\begin{array}{l}\text { Type } 2 \text { World Health Organization } \\
\text { (WHO) criteria, known type } 2 \text { and } \\
\text { using anti-diabetic medications } \\
\text { or diet }\end{array}$ & Depression & CES-D $\geq 16$ \\
\hline Lee et al., 2014 [33] & South Korea & Cross-sectional & KNHANES dataset & 9159 & $\geq 40$ & & Not specified & Depression & Single question \\
\hline Ceretta et al., 2012 [32] & Brazil & Cross-sectional & Outpatients & $\begin{array}{l}994 \text { cases and } 2145 \\
\text { controls }\end{array}$ & $>18$ & & Type $2>5$ years. On insulin $>1$ year. & $\begin{array}{l}\text { Depression } \\
\text { and SI }\end{array}$ & MINI \\
\hline
\end{tabular}


Table 2. Data from selected studies for systematic review and meta-analysis (depression studies)

\begin{tabular}{|c|c|c|c|c|c|c|}
\hline Author \& Year & Outcome & $\begin{array}{l}\text { Total Number of } \\
\text { Diabetic Patients }\end{array}$ & Number of Diabetic Events & Reported Estimate $(95 \% \mathrm{CI})$ & Adjusted Estimate $(95 \% \mathrm{CI})$ & Adjustments \\
\hline Icks et al., 2013 [43] & $\begin{array}{c}\text { Depression } \\
\text { (diagnosed diabetics) }\end{array}$ & 255 & 18 & & $1(0.59-1.68)$ & $\begin{array}{c}\text { age and sex, body mass index (BMI), } \\
\text { myocardial infarction (MI), stroke, } \\
\text { physical activity, education }\end{array}$ \\
\hline Cleal et al., 2017 [36] & Depression & 98,006 & 19,849 & & & \\
\hline \multirow[t]{2}{*}{ O'Connor et al., 2009 [34] } & \multirow[t]{2}{*}{ Depression } & $\begin{array}{c}\text { Prevalent diabetes } \\
14,144\end{array}$ & 1117 & & \multirow{2}{*}{$\begin{array}{l}\text { For subjects with low physician visits } \\
\qquad \mathrm{OR}=1.46(1.19-1.8)\end{array}$} & \multirow[t]{2}{*}{ Age, sex, number of primary care visits } \\
\hline & & Incident diabetes 2932 & 276 & & & \\
\hline Chen et al., 2013 [38] & Depression & 16,957 & 713 & & $\begin{array}{c}\text { Hazard Ratio }(\mathrm{HR})=1.43 \\
(1.16-1.77)\end{array}$ & $\begin{array}{l}\text { Age, sex, geographic area, urbanization } \\
\text { statuses, and various comorbidities }\end{array}$ \\
\hline Hamer et al., 2011 [45] & Depression & & & & $\begin{array}{c}\text { Odds ratio }(\mathrm{OR})=1.52 \\
(1.01-2.3)\end{array}$ & $\begin{array}{l}\text { Age, baseline depressive symptoms, sex, } \\
\text { smoking, alcohol intake, social status, } \\
\text { C-reactive protein (CRP), Cholesterol, } \\
\text { and BMI }\end{array}$ \\
\hline Hsu et al., 2011 [40] & Depression & 14,048 & 258 & $\mathrm{HR}=1.79(1.54-2.07)$ & $\begin{array}{l}\mathrm{HR}=1.46 \\
(1.24-1.71)\end{array}$ & $\begin{array}{l}\text { Age, sex, occupation and income and } \\
\text { comorbidity including hypertension, } \\
\text { stroke, hyperlipidemia and coronary } \\
\text { artery disease }\end{array}$ \\
\hline Golden et al., 2008 [42] & Depression & 417 & $\begin{array}{c}\text { Incidence density } 62 / 1000 \text { for diabetic } \\
\text { patients and } 37 / 1000 \text { non diabetics } 60 \\
\text { developed depression }\end{array}$ & & $\begin{array}{l}\mathrm{OR}=1.52 \\
(1.09-2.12)\end{array}$ & $\begin{array}{c}\text { Race, ethnicity, exam site, BMI, } \\
\text { Socio-economic status (SES), lifestyle } \\
\text { factors, diabetes severity (dyslipidemia, } \\
\text { hypertension (HTN), HTN medications } \\
\text { microalbuminuria) }\end{array}$ \\
\hline Huang et al., 2012 [39] & Depression & 5685 & 331 (cumulative incidence) & $\begin{array}{c}\text { Annual prevalence for diabetics } 34 / 1000 \\
\text { for non-diabetics }=11 / 1000 \text { Cumulative } \\
\text { prevalence } 92 / 1000 \text { for diabetics and } \\
41 / 1000 \text { for non-diabetics }\end{array}$ & & \\
\hline $\begin{array}{l}\text { Demakakos et al., } \\
2014[44]\end{array}$ & Depression & & & $\begin{array}{c}\text { OR }(52-64 \text { years. })=2.17(1.33-3.56) \\
\text { OR }(>65 \text { years. })=0.96(0.59-1.57)\end{array}$ & $\begin{array}{l}\text { OR }(52-64 \text { years. })=1.83(1.06-3.18) \\
\text { OR }(>65 \text { years })=0.81(0.48-1.37)\end{array}$ & $\begin{array}{c}\text { Age, elevated depressive symptoms at } \\
\text { baseline, sex, marital status, education, } \\
\text { household wealth, cardio-vascular and } \\
\text { non cardiovascular comorbidities, BMI } \\
\text { health behavior smoking alcohol } \\
\text { consumption frequency and } \\
\text { physical activity }\end{array}$ \\
\hline Knol et al., 2009 [37] & Depression & 49,593 & 7631 & & $\begin{array}{c}\text { Relative risk }(\mathrm{RR})=1.71 \\
(1.36-2.13)\end{array}$ & Age, sex, chronic disease \\
\hline Aarts et al., 2010 [41] & Depression & 6140 & 122 & $\begin{array}{c}\mathrm{HR}=1.32 \\
(1.19-1.48)\end{array}$ & $\begin{array}{l}\mathrm{HR}=1.26 \\
(1.12-1.42)\end{array}$ & $\begin{array}{l}\text { Age, practice identification code and } \\
\text { depression preceding diabetes }\end{array}$ \\
\hline Kivimaki et al., 2010 [35] & Depression & 493 & 36 & $\begin{array}{c}\mathrm{OR}=2 \\
(1.57-2.55)\end{array}$ & & $\begin{array}{l}\text { Matching was based on } 6 \text { variables: age } \\
\text { group, sex, socioeconomic position, type } \\
\text { of employment, type of employer, and } \\
\text { geographic area workplace }\end{array}$ \\
\hline Ryu et al., 2016 [46] & Depression & 237 & 205 & & $\mathrm{OR}=2.8(1.9-4.1)$ & Educational level and obesity \\
\hline Icks et al., 2008 [43] & Depression & 352 & 47 & & $\begin{aligned} \text { OR }(\text { male }) & =0.5(0.27-0.91) \\
\text { OR (female) } & =1.14(0.73-1.76)\end{aligned}$ & $\begin{array}{l}\text { Age, co-morbidity, depression induced } \\
\text { medications, smoking, activity level, } \\
\text { living without a partner, and education }\end{array}$ \\
\hline
\end{tabular}


Table 2. Cont

\begin{tabular}{|c|c|c|c|c|c|c|}
\hline Author \& Year & Outcome & $\begin{array}{l}\text { Total Number of } \\
\text { Diabetic Patients }\end{array}$ & $\begin{array}{c}\text { Number of } \\
\text { Diabetic Events }\end{array}$ & Reported Estimate $(95 \%$ CI) & Adjusted Estimate (95\% CI) & Adjustments \\
\hline James et al., $2010[47]$ & Depression & 200 & 60 & & & \\
\hline Bruce et al., 2016 [56] & Depression & 184 & 23 & & & \\
\hline Lin et al., 2008 [48] & Depression & & & $\begin{array}{c}\mathrm{OR}=1.38 \\
(1.15-1.66)\end{array}$ & & Age and gender \\
\hline Van Doreen et al., 2016 [54] & Depression & 253 & 22 & & $\begin{array}{l}\mathrm{OR}=1.73 \\
(1.38-3.6)\end{array}$ & Age, sex and education level \\
\hline Foran et al., 2015 [57] & Depression & 283 & 62 & & & \\
\hline Chung et al., 2014 [31] & Depression & 3846 & 678 & $\begin{array}{c}\mathrm{OR}=1.376 \\
(1.258-1.504)\end{array}$ & $\begin{array}{l}\mathrm{OR}=1.178 \\
(1.07-1.297)\end{array}$ & $\begin{array}{l}\text { Age, sex, smoking, alcohol, education, income, physical activity, } \\
\text { number of chronic diseases, presence of major cancer }\end{array}$ \\
\hline $\begin{array}{l}\text { Albertorio-Diaz et al., } \\
2017[58]\end{array}$ & Depression & & & $\begin{array}{l}\mathrm{OR}(\text { minor })=2.38(1.78-3.19) \\
\mathrm{OR}(\text { major })=2.81(1.92-4.11)\end{array}$ & $\begin{array}{l}\text { OR }(\text { minor })=1.95(1.39-2.74) \\
\text { OR }(\text { major })=2.28(1.45-3.57)\end{array}$ & $\begin{array}{l}\text { Effects of age, sex, race and ethnicity, education, body mass index, } \\
\text { and poverty }\end{array}$ \\
\hline Berg et al., 2012 [53] & & 121,392 & 15,511 & & $\begin{array}{l}\mathrm{OR}=1.53 \\
(1.5-1.56)\end{array}$ & Age and gender \\
\hline Meurs et al., 2016 [49] & Depression & 1811 & 90 & & $\begin{array}{l}\mathrm{OR}=1.39 \\
(1.1-1.76)\end{array}$ & Age, sex, added comorbidity and anxiety disorders \\
\hline Mantyselka et al., 2011 [59] & Depression & & & & $\begin{array}{l}\mathrm{OR}(>10)=1.35(0.84-2.15) \\
\mathrm{OR}(>16)=1.56(0.65-3.5)\end{array}$ & Demographic, lifestyle, and biological factors \\
\hline Clarke et al., 2016 [50] & Depression & 913 & 130 & & & \\
\hline Bouwman et al., 2010 [60] & Depression & 181 & 38 & $\mathrm{OR}=1.86(1.27-2.72)$ & $\begin{array}{l}\mathrm{OR}=1.77 \\
(1.13-2.78)\end{array}$ & $\begin{array}{l}\text { Age, education, family history of diabetes, triglycerides, high } \\
\text { density lipoproteins (HDL) cholesterol, total Cholesterol, } \\
\text { hypertension, smoking and waist circumference }\end{array}$ \\
\hline Li et al., 2016 [61] & Depression & 529 & 40 & & $\begin{array}{c}\mathrm{OR}=1.7 \\
(1.25-2.31)\end{array}$ & $\begin{array}{l}\text { Age, sex, and race, education level, family income, marital status, } \\
\text { and family history of diabetes body mass index, diet score, sleep } \\
\text { duration, current smoking, drinking status, and physical activity } \\
\text { history of chronic disease, and any medication }\end{array}$ \\
\hline Seglam et al., 2010 [62] & Depression & 500 & 169 & & & \\
\hline Kim et al., 2015 [63] & Depression & 175 & 41 & $\begin{array}{l}\mathrm{OR}=2.24 \\
(1.43-3.51)\end{array}$ & $\begin{array}{l}\mathrm{OR}=1.65 \\
(0.93-2.92)\end{array}$ & $\begin{array}{l}\text { Age, education, race/ethnicity, marital status, ratio of family income } \\
\text { to poverty, physical activity, BMI, and waist circumference were } \\
\text { controlled. }\end{array}$ \\
\hline Islam et al., 2015 [64] & Depression & 591 & 100 & & $\begin{array}{l}\mathrm{OR}=6.4 \\
(3.4-12.3)\end{array}$ & $\begin{array}{l}\text { Education, age occupation, marital status, BMI, HTN, no of } \\
\text { complications }\end{array}$ \\
\hline Wiltink et al., 2014 [65] & Depression & 1074 & 107 & & & \\
\hline Bessel et al., 2016 [51] & Depression & 1096 & 63 & & $\begin{array}{c}\text { (Prevalence ratio) PR }=1.31 \\
(0.97-1.78)\end{array}$ & $\begin{array}{l}\text { Sex, age, race, marital status and smoking, physical activity, body } \\
\text { mass index and waist-hip ratio. }\end{array}$ \\
\hline Adriaanse et al., 2008 [66] & Depression & 126 & 22 & $\begin{aligned} \text { OR (male) } & =2.04(0.76-5.49) \\
\text { OR (female) } & =3.18(1.31-7.74)\end{aligned}$ & $\begin{array}{l}\text { OR (male) }=1.52(0.47-4.94) \\
\text { OR (female) }=2.76(1.01-7.5)\end{array}$ & $\begin{array}{l}\text { Age, low education and diabetes symptoms (hyperglycemic, } \\
\text { cardiovascular, neuropathic pain, sensibility and ophthalmological) }\end{array}$ \\
\hline Westra et al., 2016 [67] & Depression & & & $\begin{array}{l}\mathrm{OR}=3.04 \\
(1.57-5.88)\end{array}$ & $\begin{array}{l}\mathrm{OR}=1.98 \\
(0.95-4.12)\end{array}$ & $\begin{array}{l}\text { Age, total body fat percentage, physical activity, education level, } \\
\text { time of blood/CES-D collection, serum 25-hydroxyvitamin D, sex }\end{array}$ \\
\hline Lee et al., 2014 [33] & Depression & 811 & 152 & & & \\
\hline Ceretta et al., 2012 [32] & Depression & 996 & 664 & $\mathrm{OR}=6.5(5.4-7.5)$ & $\begin{array}{c}\mathrm{OR}=1.8 \\
(1.7-2)\end{array}$ & \\
\hline
\end{tabular}


Table 3. Characteristics of selected suicidality studies.

\begin{tabular}{|c|c|c|c|c|c|c|c|c|c|}
\hline Author \& Year & Country & Study Design & Setting & Patients $N$ & Age & Female \% & Type of Diabetes & Outcome Reported & $\begin{array}{l}\text { Evaluation of } \\
\text { Outcome }\end{array}$ \\
\hline Singhal et al., 2014 [68] & England & $\begin{array}{l}\text { Retrospective } \\
\text { Cohort }\end{array}$ & $\begin{array}{l}\text { Hospital day cases or } \\
\text { inpatients }\end{array}$ & $\begin{array}{c}\text { 2,230,207 diabetic } \\
\text { patient }\end{array}$ & $\geq 10$ & - & Not specified (Hospital records) & Self-Harm \& Suicide & $\begin{array}{c}\text { Record } \\
\text { linkage/ICD-10 }\end{array}$ \\
\hline Webb et al., 2012 [69] & UK & $\begin{array}{l}\text { Nested Case } \\
\text { Control }\end{array}$ & $\begin{array}{l}\text { General practice } \\
\text { research database }\end{array}$ & 48,426 & $17-87$ & 45.4 & Not specified (ICD-9) & Self-Harm & ICD-9 \\
\hline Myers et al., 2013 [70] & USA & Cross Sectional & Outpatients & 145 & $18-75$ & 59.3 & Type 2 (self- reported) & Suicide attempt & Self- reported \\
\hline $\begin{array}{l}\text { Radobuljac et al., } \\
2009[25]\end{array}$ & Slovenia & Cross Sectional & & 625 & $14-19$ & 59 & Type 1 (record data) & $\begin{array}{l}\text { Suicidal ideation \& } \\
\text { attempt }\end{array}$ & Self-reported \\
\hline Lee et al., 2014 [33] & Korea & Cross Sectional & KNHANES data V & 8322 & $\geq 40$ & - & $\begin{array}{l}\text { Not specified (self- reported physician } \\
\text { diagnosis) }\end{array}$ & Suicidal ideation & Self-reported \\
\hline Chung et al., 2014 [31] & Korea & Cross Sectional & KNHANES data IV, $\mathrm{V}$ & 34,056 & $\geq 20$ & 57 & $\begin{array}{l}\text { Not specified (self- reported physician } \\
\text { diagnosis) }\end{array}$ & $\begin{array}{l}\text { Suicidal ideation \& } \\
\text { attempt }\end{array}$ & CIDI-SF \\
\hline Han et al., 2013 [23] & Korea & Cross Sectional & KNHANES data IV & 17,065 & $\geq 20$ & 57.6 & $\begin{array}{l}\text { Not specified (self- reported physician } \\
\text { diagnosis) }\end{array}$ & Suicidal ideation & Self-reported \\
\hline Igwe et al., 2013 [71] & Nigeria & Cross Sectional & $\begin{array}{c}\text { Outpatient } \\
\text { endocrinology clinic }\end{array}$ & 270 & $\begin{array}{l}\text { 18-64 mean: } \\
51 \pm 10.1\end{array}$ & 64.3 & $\begin{array}{l}\text { Type } 1 \text { \& Type } 2 \text { at least one year after } \\
\text { diagnosis (consultant diagnosis) }\end{array}$ & Suicidal ideation & MINI \\
\hline Handley et al., 2016 [72] & Australia & Cross Sectional & $\begin{array}{l}\text { Diabetes MILES } \\
\text { national survey }\end{array}$ & 3338 & $\begin{array}{l}\text { 18-70 Mean: } \\
51.7(13.8)\end{array}$ & 53.8 & $\begin{array}{c}\text { Type } 1 \& \text { Type } 2 \text { (the National } \\
\text { Diabetes Services Scheme Register) }\end{array}$ & Suicidal ideation & PHQ-9 (item 9) \\
\hline Ceretta et al., 2012 [32] & Brazil & Cross Sectional & $\begin{array}{l}\text { Outpatients public } \\
\text { health facility }\end{array}$ & $\begin{array}{l}994 \text { cases and } 2145 \\
\text { control }\end{array}$ & $>18$ & $56.6-59.2$ & Type 2 (self-reported) & Suicidal ideation & MINI \\
\hline Sendela et al., 2015 [73] & Poland & Cross Sectional & Outpatients & 477 & $\begin{array}{l}\text { 7-18 Mean: } \\
13.1 \pm 2.7\end{array}$ & 51.3 & Type 1 & Suicidal ideation & $\begin{array}{l}\text { CDI } \\
\text { (Item 9) }\end{array}$ \\
\hline $\begin{array}{l}\text { Fuller and Sawyer, } \\
2009[74]\end{array}$ & Canada & Cross Sectional & $\begin{array}{c}\text { Canadian } \\
\text { Community Health } \\
\text { Survey (CCHS) }\end{array}$ & 82,675 & $\geq 12$ & - & $\begin{array}{l}\text { Type } 1 \text { (self-reported diagnosis and } \\
\text { Insulin within one month of } \\
\text { diagnosis) }\end{array}$ & Suicidal ideation & Self- reported \\
\hline Batty et al., 2012 [75] & Korea & Prospective Cohort & $\begin{array}{l}\text { Cancer prevention } \\
\text { study participants }\end{array}$ & $1,234,927$ & $30-95$ & - & $\begin{array}{c}\text { Not specified (self report physician } \\
\text { diagnosis or medication, study } \\
\text { detected diabetes if FBG } \geq 126 \text { with no } \\
\text { history of diabetes) }\end{array}$ & Suicide & Death Certificates \\
\hline Yamauchi et al., 2016 [52] & Japan & Prospective Cohort & & 105,408 & $51.2 \pm 7.9$ & - & $\begin{array}{l}\text { Not specified (self-report of physician } \\
\text { diagnosis or medication usage) }\end{array}$ & Suicide & $\begin{array}{c}\text { Death } \\
\text { Certificates/ICD-10 }\end{array}$ \\
\hline Webb et al., 2014 [76] & Sweden & Cohort & Data records & $\begin{array}{l}252,191 \text { cases and } \\
1,260,214 \text { controls }\end{array}$ & $\begin{array}{l}\text { Median } 69.3 \text { Inter } \\
\text { quartile range (IQR) } \\
\quad=(59.2-78.7)\end{array}$ & 44.5 & Type 1 \& Type 2 (diabetes register) & Suicide & Death Register \\
\hline Davis et al., 2015 [77] & Australia & Cohort & $\begin{array}{l}\text { Fremantle diabetes } \\
\text { study }\end{array}$ & $1413+5660$ & $\begin{array}{l}18-89.7 \text { Mean: } \\
\quad 62.3 \pm 12.7\end{array}$ & 50.2 & Not specified & Suicide & $\begin{array}{c}\text { Death Certificate or } \\
\text { coroner's } \\
\text { determination }\end{array}$ \\
\hline Webb et al., 2012 [24] & & $\begin{array}{l}\text { Nested Case } \\
\text { Control }\end{array}$ & $\begin{array}{c}\text { Primary care } \\
\text { longitudinal database }\end{array}$ & $\begin{array}{l}473 \text { cases } 17,460 \\
\text { controls }\end{array}$ & 17-87 Median: 38 & - & Not specified (ICD-9) & Suicide & $\begin{array}{l}\text { ICD-10/ data } \\
\text { linkage }\end{array}$ \\
\hline
\end{tabular}


Table 4. Data from selected suicidality studies for systematic review and meta-analysis (suicidality studies).

\begin{tabular}{|c|c|c|c|c|c|c|}
\hline Author \& Year & Outcome & $\begin{array}{l}\text { Total Number of } \\
\text { Diabetic Patients }\end{array}$ & $\begin{array}{l}\text { Number of Diabetic } \\
\text { Events }\end{array}$ & $\begin{array}{c}\text { Reported Estimate } \\
(95 \% \text { CI })\end{array}$ & Adjusted Estimate $(95 \% \mathrm{CI})$ & Adjustments \\
\hline \multirow{2}{*}{ Singhal et al., 2014 [68] } & $\begin{array}{l}\text { Suicidal Attempt } \\
\text { (SA) }\end{array}$ & $2,230,207$ & 12,433 & $\begin{array}{c}\text { Rate ratio }(\mathrm{RR}) \\
(1.5-1.6)\end{array}$ & - & - \\
\hline & Suicide & $2,230,207$ & 626 & $\begin{array}{c}\mathrm{RR}=1 \\
(0.9-1.1)\end{array}$ & - & - \\
\hline Webb et al., 2012 [69] & Self-Harm & & 81 & $\begin{array}{c}\text { Odds ratio }(\mathrm{OR})=1.62 \\
(1.28-2.06)\end{array}$ & $\begin{array}{l}\mathrm{OR}=1.28 \\
(1-1.64)\end{array}$ & Clinical depression \\
\hline Myers et al., 2013 [70] & SA & 145 & 14 & - & - & - \\
\hline \multirow{3}{*}{ Radobuljc et al., 2009 [25] } & $\mathrm{SA}$ & 126 & 11 & - & - & - \\
\hline & Self-Harm & 126 & 16 & - & - & - \\
\hline & Suicidal Ideation (SI) & 126 & 45 & - & - & - \\
\hline Lee et al., 2014 [33] & SI & 811 & 187 & & $\begin{array}{l}\mathrm{OR}=1.24 \\
(0.95-1.61)\end{array}$ & $\begin{array}{l}\text { Age, sex, marital status, educational level, co-morbidities, } \\
\text { depressive symptoms, stress }\end{array}$ \\
\hline \multirow{2}{*}{ Chung et al., 2014 [31] } & $\mathrm{SA}$ & 3846 & 49 & $\begin{array}{l}\mathrm{OR}=1.562 \\
(1.48-2.13)\end{array}$ & $\begin{array}{l}\mathrm{OR}=1.413 \\
(1.02-1.96)\end{array}$ & $\begin{array}{l}\text { Age, sex, smoking, alcohol, education, income, physical } \\
\text { activity, number of chronic diseases and presence of major } \\
\text { cancer }\end{array}$ \\
\hline & SI & 3846 & 796 & $\begin{array}{l}\mathrm{OR}=1.481 \\
(1.36-1.61)\end{array}$ & $\begin{array}{c}1.15 \\
(1.05-1.26)\end{array}$ & $\begin{array}{l}\text { Age, sex, smoking, alcohol, education, income, physical } \\
\text { activity, number of chronic diseases and presence of major } \\
\text { cancer }\end{array}$ \\
\hline Han et al., 2013 [23] & SI & 1110 & 206 & & $\begin{array}{l}\mathrm{OR}=1.24 \\
(1.02-1.51)\end{array}$ & $\begin{array}{l}\text { Age, sex, body mass index, household income, educational } \\
\text { level, marital status, smoking, alcohol, and other chronic }\end{array}$ \\
\hline Igwe et al., 2013 [71] & SI & 270 & 17 & & & \\
\hline Handley et al., 2016 [72] & SI & 3338 & 477 & & & \\
\hline Ceretta et al., 2012 [32] & SI & 996 & 131 & $\begin{array}{c}\mathrm{OR}=7.1 \\
(5-10)\end{array}$ & $\begin{array}{c}\mathrm{OR}=2 \\
(1.6-2.3)\end{array}$ & \\
\hline Sendela et al., 2015 [73] & SI & 477 & 47 & & & \\
\hline Fuller and Sawyer, 2009 [74] & SI & 190 & 31 & & $\begin{array}{l}\mathrm{OR}=1.61 \\
(1.08-2.42)\end{array}$ & Age and sex \\
\hline Batty et al., 2012 [75] & Suicide & 13,452 & 12 & & $\begin{array}{c}\text { Hazard ratio }(\mathrm{HR})(\text { male })=2.55 \\
(1.3-5), \\
\text { HR }(\text { female })=3.64 \\
(1.12-11.86)\end{array}$ & $\begin{array}{l}\text { Exercise, smoking status, alcohol consumption, body mass } \\
\text { index, height, blood pressure and blood cholesterol. }\end{array}$ \\
\hline Yamauchi et al., 2016 [52] & Suicide & 4898 & 41 & & $\begin{array}{c}\text { OR }(\text { male })=1.2 \\
(0.9-1.8) \\
\text { OR (female) }=1.5(0.7-3)\end{array}$ & $\begin{array}{l}\text { Age at study entry, public health center area, smoking } \\
\text { status, alcohol-drinking habits, body mass index } \\
\text { cohabitation, employment status, hours of sleep, frequency } \\
\text { of physical exercise, stress level and history of major } \\
\text { physical illnesses }\end{array}$ \\
\hline Webb et al., 2014 [76] & Suicide & 252,191 & 482 & & $\begin{array}{l}\mathrm{RR}=3.36 \\
(2.99-3.79)\end{array}$ & Age, sex and country of birth \\
\hline Davis et al., 2015 [77] & Suicide & 1413 & 4 & & $\begin{array}{l}\mathrm{OR}=1.16 \\
(0.38-3.51)\end{array}$ & Age and sex \\
\hline Webb et al., 2012 [24] & Suicide & 892 & 47 & $\begin{array}{l}\mathrm{OR}=1.18 \\
(0.85-1.62)\end{array}$ & $\begin{array}{c}\mathrm{OR}=0.9 \\
(0.65-1.26)\end{array}$ & $\begin{array}{l}\text { Sex and age by the case-control matching with added } \\
\text { adjustment for clinical depression. }\end{array}$ \\
\hline
\end{tabular}


(1) Completed Suicide

Seven studies reported data on completed suicide (all cohort). Suicidal death was confirmed by either International Classification of Diseases (ICD) codes or examination of death certificate.

(2) Suicidal Attempts

Five studies assessed suicidal attempts and self-harm (one cohort, one nested case-control and three cross-sectional). Attempted suicide was evaluated based on either ICD codes or self-reported information.

(3) Suicidal Ideation

Nine cross-sectional studies examined suicidal ideation. Three of them evaluated type 1 diabetics, one study evaluated type 2 diabetic patients, who were on insulin [32], while the remaining studies included both type 1 and type 2 patients. Two studies had minors as participants (adolescents) [25,73]. All studies used self-reported information to evaluate for the presence of suicidal ideation either as a response to a single question, or as a part of depression screening questionnaires, or during a standardized interview.

\subsection{Main Meta-Analysis Results}

\subsubsection{Depression}

Depression results are reported as odds ratios (adjusted and unadjusted) by study design. The overall OR based on all depression studies (cohort and cross-sectional) was 1.79 (95\% CI: 1.62-1.99), with significant heterogeneity $\left(I^{2}=98.09 \%, Q=9.66\right.$, and $p$-value $\left.<0.001\right)$.

(1) Cohort Studies

The pooled unadjusted odds ratio for the association between diabetes and depression calculated based on all 13 studies, using a random effect model was 1.49 (95\% CI: 1.36-1.64, $p$-value $<0.001$ ). Forest plot of the OR and 95\% CI for the random effect model are shown in Figure 2. There was significant heterogeneity between studies $\left(I^{2}=94.08 \%, Q=203.12, p\right.$-value $\left.<0.001\right)$. The pooled adjusted effect estimate was calculated based on five studies [35,42-45] with an OR $=1.48(95 \% \mathrm{CI}$ : $1.16-1.88)$ and $p$-value $=0.001$. The test for heterogeneity was not significant $\left(I^{2}=54.2 \%, Q=8.73\right.$, $p$-value $=0.068)$.

(2) Cross-Sectional Studies

Unadjusted odds ratio for depression among diabetics, calculated based on cross-sectional studies was $2.04(95 \%$ CI: 1.73-2.42) $p$-value $<0.001$. However, there was a significant heterogeneity $\left(I^{2}=93.79 \%, Q=370.7, p\right.$-value $\left.<0.001\right)$. The adjusted odds ratio from cross-sectional studies was 1.67 (95\% CI: $1.47-1.90) p$-value $<0.001$, with significant heterogeneity $\left(I^{2}=74.16 \%, Q=58.05\right.$, $p$-value $<0.001)$. 
Studyname

Chen et al, 2013
Huang et al, 2012
O'Connor et al, 2009
O'Connor et al, 2009
Hsu et al, 2011
Aarts et al, 2010
Golden et al, 2008
Icks et al, 2013
Demakakos et al, 2014
Kivimaki et al, 2010
Cleal et al, 2017
Knol et al, 2009
Hamer et al, 2011

\begin{tabular}{|c|c|c|c|}
\hline \multicolumn{4}{|c|}{ Statistics for each study } \\
\hline $\begin{array}{l}\text { Odds } \\
\text { ratio }\end{array}$ & $\begin{array}{l}\text { Lower } \\
\text { limit }\end{array}$ & $\begin{array}{l}\text { Upper } \\
\text { limit }\end{array}$ & $\mathrm{p}$-Value \\
\hline 1.327 & 1.184 & 1.487 & 0.000 \\
\hline 2.082 & 1.858 & 2.335 & 0.000 \\
\hline 1.473 & 1.340 & 1.619 & 0.000 \\
\hline 1.721 & 1.410 & 2.100 & 0.000 \\
\hline 1.800 & 1.552 & 2.088 & 0.000 \\
\hline 1.245 & 1.006 & 1.541 & 0.044 \\
\hline 1.267 & 0.942 & 1.703 & 0.118 \\
\hline 1.092 & 0.663 & 1.800 & 0.729 \\
\hline 1.440 & 1.018 & 2.037 & 0.040 \\
\hline 1.965 & 1.446 & 2.671 & 0.000 \\
\hline 1.198 & 1.179 & 1.217 & 0.000 \\
\hline 1.373 & 1.334 & 1.413 & 0.000 \\
\hline 1.769 & 1.268 & 2.469 & 0.001 \\
\hline 1.497 & 1.362 & 1.645 & 0.000 \\
\hline
\end{tabular}

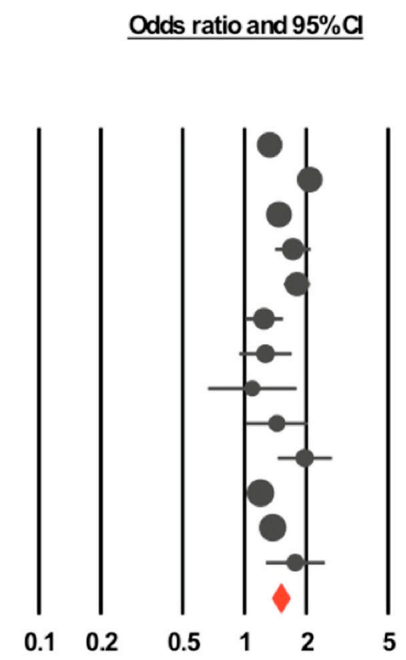

Figure 2. Forest plot for the association between depression and diabetes from cohort studies. Estimates are in the center of the box and lines represent $95 \%$ confidence intervals (CI). Diamond shows the pooled odds ratio size and its $95 \% \mathrm{CI}$.

\subsubsection{Suicidality}

Suicidality results are reported as prevalence and odds ratios (adjusted and unadjusted) stratified by outcome.

\section{(1) Completed Suicide}

Overall prevalence was $0.3 \%$ (95\% CI: $0.1-0.7 \%$ ). Based on the five included studies [55-59], the unadjusted pooled odds ratio was $1.85(95 \%$ CI: 0.97-3.52, $p$-value $=0.061)$ (Figure 2), with significant heterogeneity $\left(I^{2}=94.79 \%, Q=76.88, p\right.$-value $\left.<0.001\right)$. Adjusted OR (based on three studies) was 1.39 $(95 \%$ CI: $0.82-2.35, p$-value $=0.225)\left(I^{2}=81.66 \%, Q=10.90, p\right.$-value $\left.=0.004\right)$.

\section{(2) Suicidal Attempts}

The pooled prevalence of attempted suicide was 2.7\% (95\% CI: 0.9-7.8\%). Three studies reported unadjusted odds ratios. The calculated unadjusted pooled odds ratio was 1.45 (95\% CI: 1.07-1.96, $p$-value $=0.017)($ Figure 2$)$, with significant heterogeneity $\left(I^{2}=53.43 \%, Q=4.29, p\right.$-value $\left.=0.117\right)$. Adjusted odds ratio based on two studies [31,69] was 1.33 (95\% CI: $1.09-1.62, p$-value $=0.005)\left(I^{2}=0 \%\right.$, $Q=0.23, p$-value $=0.635$ ).

\section{(3) Suicidal Ideation}

The prevalence of suicidal ideation, among diabetics was 16.2\% (95\% CI: 8.5-28.5\%). Unadjusted pooled odds ratio (based on six studies) was 1.89 (95\% CI: 1.36-2.63, $p$-value < 0.001) (Figure 2), with significant heterogeneity $\left(I^{2}=94.09 \%, Q=84.65, p\right.$-value $\left.<0.0001\right)$. Adjusted odds ratio (based on four studies) was 1.49 (95\% CI: $1.14-1.96, p$-value $=0.004)\left(I^{2}=80.37 \%, Q=15.28, p\right.$-value $\left.=0.002\right)$.

\subsection{Subgroup Analysis and Meta-Regression}

\subsubsection{Depression}

\section{(1) Cohort Studies}

In the six studies that evaluated depression among diabetics using clinical diagnostic criteria, the pooled OR was 1.59 (95\% CI: 1.35-1.86) and the test of heterogeneity was significant $\left(I^{2}=88.09 \%\right.$, $Q=41.98, p$-value $<0.001)$. In the four studies that evaluated depression among diabetics using questionnaires, the pooled OR was 1.41 (95\% CI: 1.17-1.69), while the test of heterogeneity was not significant $\left(I^{2}=9.24 \%, Q=3.30, p\right.$-value $\left.=0.340\right)$. For the three studies that used antidepressants, 
the pooled OR was 1.36 (95\% CI: $1.19-1.54)$ and test of heterogeneity was significant $\left(I^{2}=97.32 \%\right.$, $Q=74.52$, $p$-value 0.001). Studies were stratified on the basis of assessing patients with incident diabetes $\mathrm{OR}=1.49$ (95\% CI: 1.33-1.69) and prevalent diabetes OR $=1.47$ (95\% CI: $1.25-1.72$ ). Other confounders (level of adjustment, comparison group, risk of bias, and geographical location) did not significantly impact the effect estimate. Results for meta-regression are included in Table 5.

Table 5. Meta-regression for depression cohort studies.

\begin{tabular}{|c|c|c|c|c|c|c|c|}
\hline & \multirow{2}{*}{ Reference Group } & \multirow{2}{*}{ Category } & \multirow{2}{*}{ Coefficient } & \multicolumn{2}{|c|}{$95 \% \mathrm{CI}$} & \multirow{2}{*}{$p$-Value } & \multirow{2}{*}{$R^{2}$} \\
\hline & & & & Lower & Upper & & \\
\hline $\begin{array}{l}\text { Depression } \\
\text { Evaluation }\end{array}$ & $\begin{array}{l}\text { Depressive } \\
\text { Symptoms }\end{array}$ & Anti-depressants & -0.026 & -0.283 & 0.230 & 0.218 & 0.34 \\
\hline \multirow{2}{*}{$\begin{array}{c}\text { Level of } \\
\text { Adjustment }\end{array}$} & \multirow{2}{*}{ No } & Full $(>5)$ & 0.299 & -0.126 & 0.726 & \multirow{2}{*}{0.386} & \multirow{2}{*}{0.1} \\
\hline & & Partial $(<5)$ & 0.024 & -0.162 & 0.212 & & \\
\hline Diabetes & $\begin{array}{l}\text { Prevalent } \\
\text { Diabetes }\end{array}$ & Incident Diabetes & -0.014 & -0.191 & 0.162 & 0.874 & 0.18 \\
\hline \multirow{2}{*}{$\begin{array}{c}\text { Geographical } \\
\text { location }\end{array}$} & \multirow[b]{2}{*}{ North America } & Asia & 0.083 & -0.155 & 0.032 & \multirow[b]{2}{*}{0.111} & \multirow[b]{2}{*}{0.36} \\
\hline & & Scandinavian & -0.137 & -0.375 & 0.101 & & \\
\hline
\end{tabular}

$R^{2}$ reflects the amount of variability in $I^{2}$ that is explained by the model. ${ }^{*}$ Adjusted for $<5$ confounding factors was considered partial, $>5$ was considered full.

\section{(2) Cross-Sectional Studies}

In evaluating depression, the same pattern observed for cohort studies was maintained in cross-sectional studies. In examining the differences between groups, depressive disorders ( $\mathrm{OR}=2.24$, 95\% CI: 1.46-3.45), depressive symptoms (OR $=1.47,95 \%$ CI: $1.37-1.57$ ), and antidepressants ( $\mathrm{OR}=1.89$, $95 \%$ CI: $1.86-1.92)$ were not statistically significant $(p$-value $=0.738)$.

\subsubsection{Suicidality}

The results for suicidality subgroup analysis are included in Table 6, Figure 3. Due to the limited number of studies assessing attempted suicide, no subgroup analysis was conducted.

Table 6. Results for subgroup analysis for suicide and suicidal ideations.

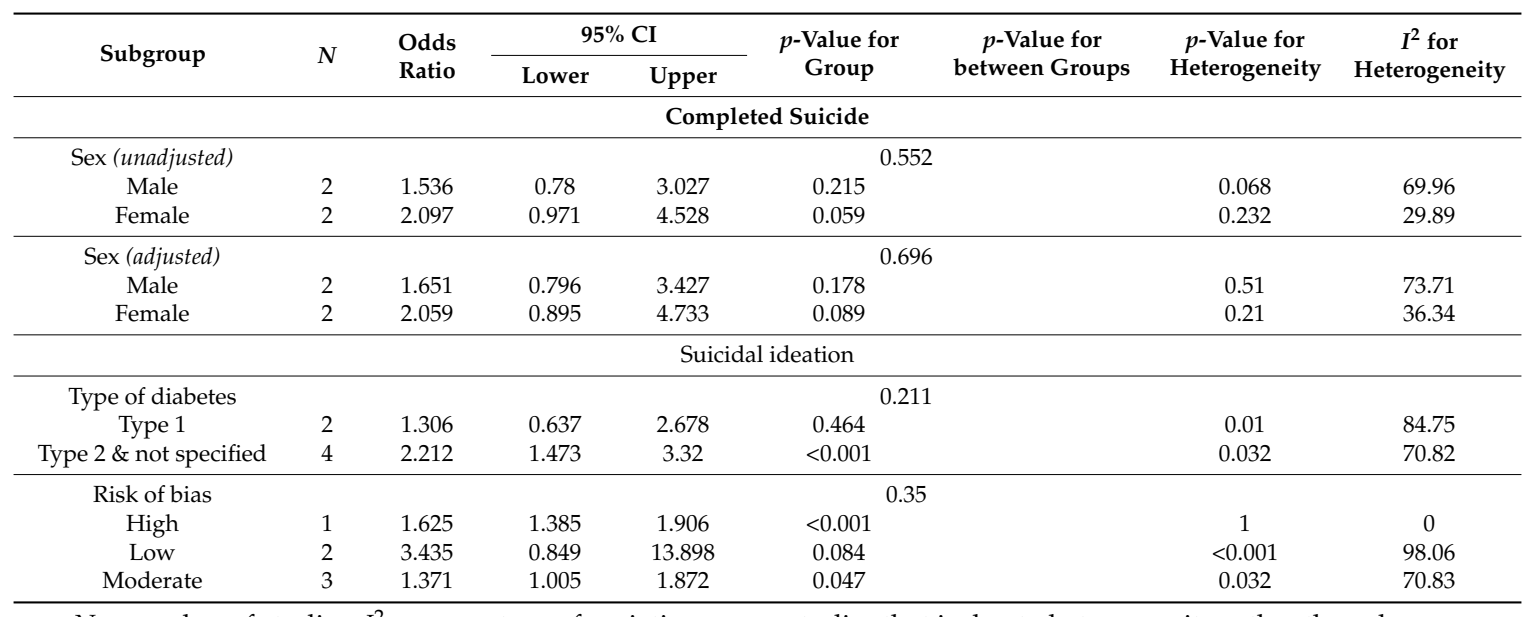

$N=$ number of studies. $I^{2}=$ percentage of variation across studies that is due to heterogeneity rather than chance. 


\begin{tabular}{|c|c|c|c|c|c|}
\hline \multirow[t]{2}{*}{ Study name } & \multirow[t]{2}{*}{ Outcome } & \multicolumn{4}{|c|}{ Statistics for each study } \\
\hline & & $\begin{array}{l}\text { Odds } \\
\text { ratio }\end{array}$ & $\begin{array}{c}\text { Lower } \\
\text { limit }\end{array}$ & $\begin{array}{l}\text { Upper } \\
\text { limit }\end{array}$ & p-Value \\
\hline Chung et al, 2014 & S. A. & 1.528 & 1.124 & 2.079 & 0.007 \\
\hline Radobuljac et al, 2009 & S. A. & 0.788 & 0.399 & 1.556 & 0.493 \\
\hline \multirow[t]{2}{*}{ Webb et al, 2012} & S.A. & 1.689 & 1.329 & 2.146 & 0.000 \\
\hline & & 1.447 & 1.069 & 1.959 & 0.017 \\
\hline Ceretta et al, 2012 & S. I. & 7.067 & 4.991 & 10.007 & 0.000 \\
\hline Chung et al, 2014 & S. I. & 1.441 & 1.325 & 1.568 & 0.000 \\
\hline Fuller and Sawyer, 2009 & S. I. & 1.879 & 1.278 & 2.763 & 0.001 \\
\hline Han et al, 2013 & S. I. & 1.625 & 1.385 & 1.906 & 0.000 \\
\hline Lee et al, 2014 & S. I. & 1.698 & 1.425 & 2.023 & 0.000 \\
\hline \multirow[t]{2}{*}{ Radobuljac et al, 2009} & S. I. & 0.904 & 0.602 & 1.357 & 0.625 \\
\hline & & 1.889 & 1.357 & 2.631 & 0.000 \\
\hline Batty et al, 2012 & Suicide & 2.585 & 1.453 & 4.599 & 0.001 \\
\hline Davis et al, 2015 & Suicide & 1.145 & 0.376 & 3.483 & 0.812 \\
\hline Webb et al, 2012 & Suicide & 1.119 & 0.827 & 1.513 & 0.466 \\
\hline Webb et al, 2014 & Suicide & 3.745 & 3.328 & 4.215 & 0.000 \\
\hline \multirow[t]{3}{*}{ Yamauchi et al, 2016} & Suicide & 1.462 & 1.064 & 2.010 & 0.019 \\
\hline & & 1.849 & 0.971 & 3.519 & 0.061 \\
\hline & & 1.656 & 1.341 & 2.045 & 0.000 \\
\hline
\end{tabular}

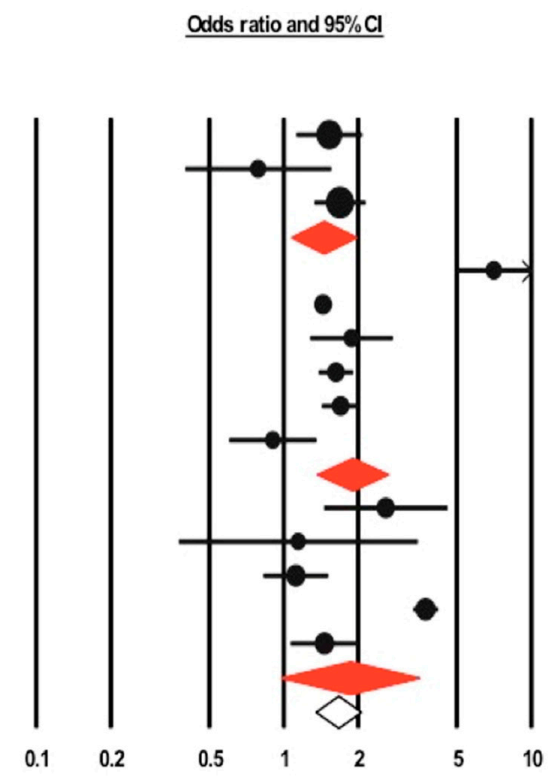

Figure 3. Forest plot of unadjusted odds ratio for suicidality and diabetes. SA: suicidal attempts, SI: suicidal ideation. Red diamonds represent pooled odds ratio for each outcome, while white diamond represent the overall odds ratio for the three outcomes.

\subsection{Influential Analysis and Publication Bias}

Influential analysis revealed that no single study had a substantial influence on either the adjusted or unadjusted effect estimates. There was evidence of publication bias for depression among cohort studies based on the inspection of the funnel plot (Figure 4) and Egger's test ( $p$-value $=0.027$ ). To account for this bias, a Duval and Tweedie test was used and reported an adjusted OR 1.25 (95\% CI: 1.15-1.37). There was no evidence of publication bias for depression among cross-sectional studies $(p$-value $=0.721)$ and suicidality studies $(p$-value $=0.860)$.

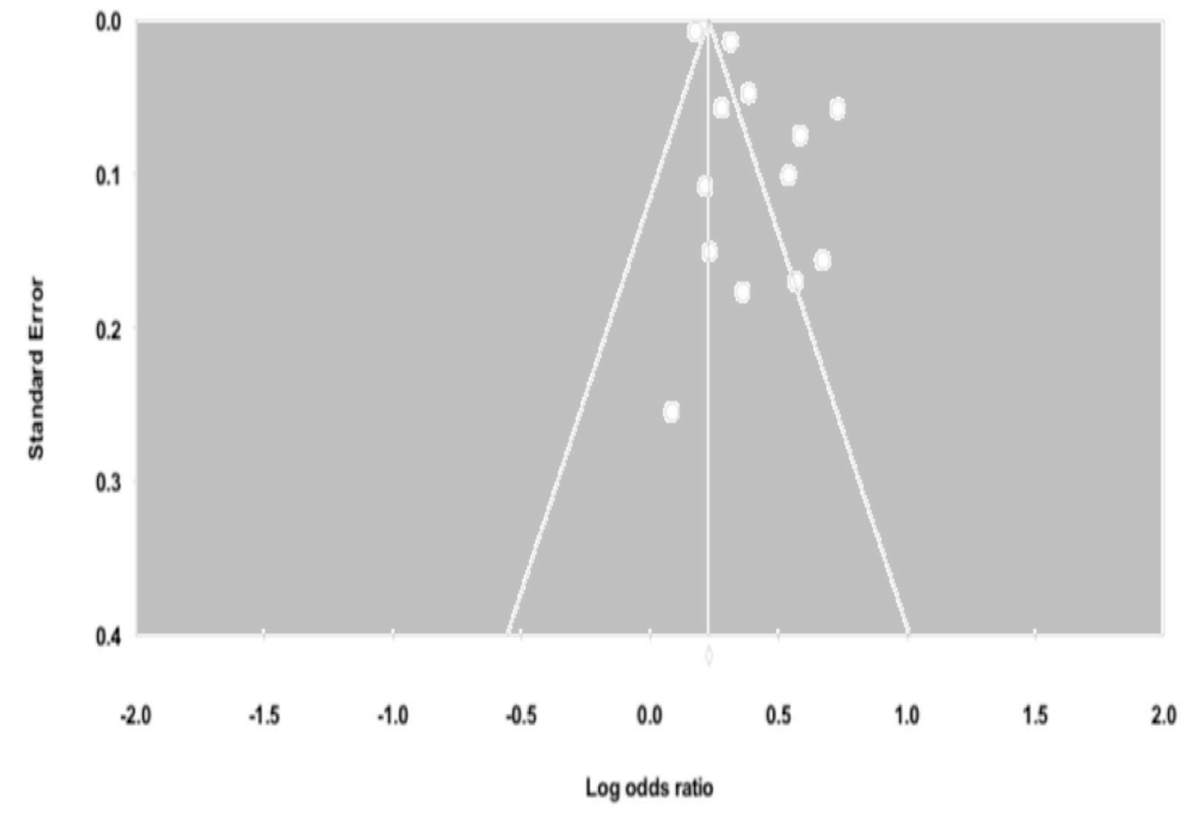

Figure 4. Funnel plots of the meta-analysis of published studies. Each point represents the log odds ratio and the standard error for a single study. The triangle represents the region where $95 \%$ of the data points would lie in the absence of a publication bias. The vertical line represents the average log odds ratio found in the meta-analysis. 


\section{Discussion}

To our knowledge, this study is one of the first meta-analyses that reports the prevalence of suicidality among diabetic patients and assesses the association between diabetes, depression, and suicidality using data from observational studies. Our results show that diabetic patients are more likely to have depression, experience suicidal ideations and attempt suicide compared to non-diabetic patients. The findings of this study help highlight diabetics as a high-risk group for depression and suicidality.

In regards to depression, based on 13 cohort studies and 23 cross-sectional studies, our results suggest that there is a significant association between depression and diabetes. It is interesting to note that this association maintained its strength even after adjusting for potential confounders. Previous systematic reviews have shown that patients diagnosed with diabetes are at higher risk for depression [7,78]. Similarly, our analysis of cohort studies corroborates the existing evidence [78] and suggests a directionality, whereby diabetes may play a causal role in the development of depression. However, the psychological burden of diabetes may lead to but does not fully account for the increased risk of depression [3]. Other potential physiological contributors include: activation of the HPA axis and SNS [11,79], chronic inflammation [80], and cerebral vascular changes induced by diabetes [81]. Additionally, some common medications used for the treatment of diabetes have been linked to a higher risk of depression $[82,83]$.

In our study, the calculated odds ratio for cross-sectional studies (assessing prevalence of depression) was higher than the one in cohort studies (assessing incidence of depression), corroborating the results reported in a previous meta-analysis [6]. This finding may be explained in part by: (1) the longer duration of depression among diabetic patients, and (2) the potential bidirectional association between diabetes and depression. The longer duration of depression among diabetic patients may be due to the increased likelihood to experience treatment resistant and recurrent forms of depression [20]. This leads to a buildup of chronic cases. The potential bidirectional association between diabetes and depression has also been examined in the literature [42,84]. Depression has been linked to the development of type 2 diabetes with the use of certain antidepressants, which are known to have clinical effects on glucose homeostasis and weight gain [85]. Additionally, depression may have a negative effect on a patient's lifestyle choices including physical activity, leading to an increased risk to develop diabetes [86]. Thus, it is unsurprising that the risk of prevalent depression was higher than that of incident depression among diabetic patients in our study.

Suicidality among diabetic patients has not been fully elucidated. Our study examined the prevalence and risk of suicidality among diabetic patients in order to address the existing gap in the literature. In the general population, a study involving 17 countries found the lifetime prevalence of suicidal ideation and attempts to be at $9.2 \%$ and $2.7 \%$, respectively [87]. By comparison, our study found that the prevalence of suicidal ideation among patients with diabetes was much higher, at a reported $16.2 \%$ (95\% CI: 8.5-28.5\%), while the rate of attempted suicide was similar, at $2.7 \%$ (95\% CI: $0.9-7.8 \%)$.

Depression is one of the leading risk factors for suicidality [22,88]. In our study, diabetic patients were found to be twice as likely to experience suicidal ideations compared to non-diabetics. When examining attempted suicide, diabetics were also significantly at higher risk compared to non-diabetics. These findings are concerning and help highlight the vulnerability of diabetic patients possibly progressing from suicidal ideation to attempted suicide. Despite the increased risk of suicidal ideation and attempts, diabetic patients did not experience a significant risk of suicidal death $(\mathrm{OR}=1.85,95 \% \mathrm{CI}$ : $0.97-3.52, p$-value $=0.061$ ) . This could be attributed to several reasons connected to suicide, including: (1) stigmatization; (2) misclassification; (3) low occurrence; (4) limited details and number of studies; and (5) lack of research into the distinct predictive factors for suicidal death.

Stigma related to suicide is a major barrier in accurately reporting and tracking of suicidal deaths [89]. The high level of stigmatization in many countries, where suicide is considered to be immoral and illegal, might lead to underreporting of suicide as a cause of death [90]. This in turn 
would negatively impact accuracy of suicide rates reported in large-scale epidemiological studies [90]. Misclassification of suicidal death due to insulin overdoses as accidental death or death due to natural causes is another possibility that need to be further evaluated. Studying suicide is also statistically challenging because of the relative low occurrence of the event and the need for large samples to obtain reliable estimates [91]. Additionally, in the present systematic review, the limited number of studies assessing suicidal attempts lacked some key details. For example, there was dearth of information assessing the seriousness of the attempt (i.e., extent of hospital care required afterward or whether the attempt resulted in permanent disabilities). Furthermore, it has been suggested in the literature that suicidal ideations and behaviors might have different predictors than completed suicide [92,93]. Therefore, we cannot fully rely on suicidal ideations to understand completed suicide. Factors that may play a role in moving from one condition to another remain unclear for diabetic patients and need to be carefully investigated.

\subsection{Strengths and Limitations}

This study has several strengths. It provides an up-to-date literature review, which includes both depression and suicidality as outcomes of interest among diabetic patients. It provides evidence that can be used as a reference point for future research focusing on specific diabetic sub-populations. Finally, it takes into consideration several important factors (i.e., method of depression evaluation, type of diabetes, whether diabetes was incident or prevalent, and geographical location) in the analysis of our data, which adds to the robustness of this study.

However, there are also several limitations we need to consider. First, there are few studies assessing suicidality, especially attempted suicide. Second, there was a marked heterogeneity among the included studies. Third, we included studies that used prescription of antidepressants as a proxy for depression. This method is quite controversial especially among diabetic patients since antidepressants are commonly used for symptomatic treatment of diabetic neuropathic pain, which may falsely increase reported estimates. However, our subgroup analysis showed that differences in depression evaluation methods did not significantly impact our results. Fourth, confounding bias could not be entirely eliminated. Fifth, association of diabetes and suicidal attempts and suicidal ideation do not imply causation. Finally, most studies included in our systematic review were from developed countries and therefore, the results of our analysis need to be interpreted with caution, as they may not be generalizable to the developing world.

\subsection{Implications for Future Research and Clinical Practice}

There are significant gaps and opportunities for research in this important public health topic. Further research is warranted to: (1) examine the factors, mechanisms and transitional triggers implicated in the association between diabetes, depression and suicidality; (2) assess the role and impact of different diabetes management strategies on the patient's risk of depression and suicidality; and (3) evaluate the cultural and ethnic differences as they relate to diabetes, depression and suicidality. Clinicians should be aware and receive cross-training in order to be better prepared to address the higher risk for depression and suicidality among diabetic patients. Additionally, several other initiatives can be considered, including: (1) early detection and treatment of depression, which would improve diabetes control and consequently, delay the development of diabetic and depression related complications; (2) the development of robust and standardized validated screening tools for diabetic patients at risk for depression and suicidality; and (3) timely design and implementation of comprehensive interventions that take into account the complex inter-relationships between depression and suicidality, so as to improve the quality of life of diabetic patients.

\section{Conclusions}

Our study found that diabetes is associated with an increased risk for depression, suicidal ideation and suicidal attempts but not completed suicide. Therefore, efforts for early detection and effective 
screening are urgently needed in primary care settings. Appropriate training for healthcare providers in the field of suicidality screening and depression management would help mitigate the negative impacts on a diabetic patient's quality of life and reduce the growing burden on healthcare systems. Given the limited number of studies on this important topic, further research is needed.

Author Contributions: J.M., R.E., Y.B., and L.T. were involved in the study conception and design. J.M. and R.E. were responsible for the data analysis. All authors contributed to the discussion, interpreted the findings, helped write, reviewed/edited the manuscript for intellectual content, and read and approved the final manuscript.

Funding: This research was supported in part by an internal grant from the School of Public of Public Health, University of Saskatchewan.

Acknowledgments: We gratefully acknowledge the support of Rita Hanoski, Health Education and Promotion Coordinator and Jocelyn Orb, Manager, Student Health Services, University of Saskatchewan.

Conflicts of Interest: The authors declare no conflict of interest.

\section{References}

1. International Diabetes Federation. IDF Diabetes, 7 Eds; International Diabetes Federation: Brussels, Belgium, 2015; Available online: http:/ / www.diabetesatlas.org (accessed on 12 May 2018).

2. World Health Organization. Global Report on Diabetes; WHO: Geneva, Switzerland, 2016.

3. Robinson, D.J.; Luthra, M.; Vallis, M. Canadian diabetes association clinical practice guidelines expert committee. Canadian diabetes association 2013 clinical practice guidelines for the prevention and management of diabetes in Canada. Can. J. Diabetes 2013, 37, S1-S212.

4. Bădescu, S.V.; Tătaru, C.; Kobylinska, L.; Georgescu, E.L.; Zahiu, D.M.; Zăgrean, A.M.; Zăgrean, L. The association between diabetes mellitus and depression. J. Med. Life 2016, 9, 120. [PubMed]

5. Katon, W.J. Epidemiology and treatment of depression in patients with chronic medical illness. Dialog. Clin. Neurosci. 2011, 13, 7.

6. Anderson, R.J.; Freedland, K.E.; Clouse, R.E.; Lustman, P.J. The prevalence of comorbid depression in adults with diabetes: A meta-analysis. Diabetes Care 2001, 24, 1069-1078. [CrossRef] [PubMed]

7. Rotella, F.; Mannucci, E. Diabetes mellitus as a risk factor for depression. A meta-analysis of longitudinal studies. Diabetes Res. Clin. Pract. 2013, 99, 98-104. [CrossRef] [PubMed]

8. Nouwen, A.; Winkley, K.; Twisk, J.; Lloyd, C.E.; Peyrot, M.; Ismail, K.; Pouwer, F. European Depression in Diabetes (EDID) Research Consortium. Type 2 diabetes mellitus as a risk factor for the onset of depression: A systematic review and meta-analysis. Diabetologia 2010, 53, 2480-2486. [CrossRef] [PubMed]

9. Samaan, Z.; Garasia, S.; Gerstein, H.C.; Engert, J.C.; Mohan, V.; Diaz, R.; Anand, S.S.; Meyre, D. Lack of association between type 2 diabetes and major depression: Epidemiologic and genetic evidence in a multiethnic population. Transl. Psychiatry 2015, 5, e618. [CrossRef] [PubMed]

10. Agardh, E.; Allebeck, P.; Hallqvist, J.; Moradi, T.; Sidorchuk, A. Type 2 diabetes incidence and socio-economic position: A systematic review and meta-analysis. Int. J. Epidemiol. 2011, 40, 804-818. [CrossRef] [PubMed]

11. Kyrou, I.; Tsigos, C. Stress hormones: Physiological stress and regulation of metabolism. Curr. Opin. Pharmacol. 2009, 9, 787-793. [CrossRef] [PubMed]

12. Nicolaides, N.C.; Kyratzi, E.; Lamprokostopoulou, A.; Chrousos, G.P.; Charmandari, E. Stress, the stress system and the role of glucocorticoids. Neuroimmunomodulation 2015, 22, 6-19. [CrossRef] [PubMed]

13. Chrousos, G.P. Stress and disorders of the stress system. Nat. Rev. Endocrinol. 2009, 5, 374-381. [CrossRef] [PubMed]

14. Moulton, C.D.; Pickup, J.C.; Ismail, K. The link between depression and diabetes: The search for shared mechanisms. Lancet Diabetes Endocrinol. 2015, 3, 461-471. [CrossRef]

15. Wang, X.; Bao, W.; Liu, J.; Ouyang, Y.Y.; Wang, D.; Rong, S.; Xiao, X.; Shan, Z.L.; Zhang, Y.; Yao, P.; et al. Inflammatory markers and risk of type 2 diabetes: A systematic review and meta-analysis. Diabetes Care 2013, 36, 166-175. [CrossRef] [PubMed]

16. Miller, A.H.; Maletic, V.; Raison, C.L. Inflammation and its discontents: The role of cytokines in the pathophysiology of major depression. Biol. Psychiatry 2009, 65, 732-741. [CrossRef] [PubMed]

17. Egede, L.E.; Nietert, P.J.; Zheng, D. Depression and all-cause and coronary heart disease mortality among adults with and without diabetes. Diabetes Care 2005, 28, 1339-1345. [CrossRef] [PubMed] 
18. Ciechanowski, P.S.; Katon, W.J.; Russo, J.E. Depression and diabetes: Impact of depressive symptoms on adherence, function, and costs. Arch. Int. Med. 2000, 160, 3278-3285. [CrossRef]

19. Lin, E.H.; Katon, W.; Von Korff, M.; Rutter, C.; Simon, G.E.; Oliver, M.; Ciechanowski, P.; Ludman, E.J.; Bush, T.; Young, B. Relationship of depression and diabetes self-care, medication adherence, and preventive care. Diabetes Care 2004, 27, 2154-2160. [CrossRef] [PubMed]

20. Peyrot, M.; Rubin, R.R. Persistence of depressive symptoms in diabetic adults. Diabetes Care 1999, $22,448-452$. [CrossRef] [PubMed]

21. World Health Organization. Suicide. Available online: http://www.who.int/mediacentre/factsheets/fs398/ en/ (accessed on 19 December 2017).

22. Klonsky, E.D.; May, A.M.; Saffer, B.Y. Suicide, suicide attempts, and suicidal ideation. Ann. Rev. Clin. Psychol. 2016, 12, 307-330. [CrossRef] [PubMed]

23. Han, S.J.; Kim, H.J.; Choi, Y.J.; Lee, K.W.; Kim, D.J. Increased risk of suicidal ideation in Korean adults with both diabetes and depression. Diabetes Res. Clin. Pract. 2013, 101, e14-e17. [CrossRef] [PubMed]

24. Webb, R.T.; Kontopantelis, E.; Doran, T.; Qin, P.; Creed, F.; Kapur, N. Suicide risk in primary care patients with major physical diseases: A case-control study. Arch. Gen. Psychiatry 2012, 69, 256-264. [CrossRef] [PubMed]

25. Radobuljac, M.D.; Bratina, N.U.; Battelino, T.; Tomori, M. Lifetime prevalence of suicidal and self-injurious behaviors in a representative cohort of Slovenian adolescents with type 1 diabetes. Pediatr. Diabetes 2009, 10, 424-431. [CrossRef] [PubMed]

26. Wells, G.A.; Shea, B.; O'Connell, D.; Peterson, J.; Welch, V.; Losos, M.; Tugwell, P. The Newcastle-Ottawa Scale (NOS) for Assessing the Quality of Non-Randomised Studies in Meta-Analyses; Ottawa Hospital Research Institute: Ottawa, ON, Canada, 2009; Available online: www.ohri.ca/programs/clinical_epidemiology/oxford.asp (accessed on 10 May 2018).

27. Borenstein, M.; Hedges, L.; Higgins, J.; Rothstein, H. Comprehensive Meta-Analysis; Biostat Inc.: Englewood, NJ, USA, 2014.

28. DerSimonian, R.; Laird, N. Meta-analysis in clinical trials. Controlled Clinical Trials. 1986, 7, 177-188. [CrossRef]

29. Egger, M.; Smith, G.D.; Schneider, M.; Minder, C. Bias in meta-analysis detected by a simple, graphical test. BMJ 1997, 315, 629-634. [CrossRef] [PubMed]

30. Duval, S.; Tweedie, R. A nonparametric "trim and fill" method of accounting for publication bias in meta-analysis. J. Am. Stat. Assoc. 2000, 95, 89-98.

31. Chung, J.H.; Moon, K.; Kim, D.H.; Min, J.W.; Kim, T.H.; Hwang, H.J. Suicidal ideation and suicide attempts among diabetes mellitus: The Korea National Health and Nutrition Examination Survey (KNHANES IV, V.) from 2007 to 2012. J. Psychosom. Res. 2014, 77, 457-461. [CrossRef] [PubMed]

32. Ceretta, L.B.; Réus, G.Z.; Abelaira, H.M.; Jornada, L.K.; Schwalm, M.T.; Hoepers, N.J.; Tomazzi, C.D.; Gulbis, K.G.; Ceretta, R.A.; Quevedo, J. Increased prevalence of mood disorders and suicidal ideation in type 2 diabetic patients. Acta Diabetol. 2012, 49, 227-234. [CrossRef] [PubMed]

33. Lee, H.Y.; Hahm, M.I.; Lee, S.G. Risk of suicidal ideation in diabetes varies by diabetes regimen, diabetes duration, and HbA1c level. J. Psychosom. Res. 2014, 76, 275-279. [CrossRef] [PubMed]

34. O'Connor, P.J.; Crain, A.L.; Rush, W.A.; Hanson, A.M.; Fischer, L.R.; Kluznik, J.C. Does diabetes double the risk of depression? Ann. Family Med. 2009, 7, 328-335. [CrossRef] [PubMed]

35. Kivimäki, M.; Tabák, A.G.; Lawlor, D.A.; Batty, G.D.; Singh-Manoux, A.; Jokela, M.; Virtanen, M.; Salo, P.; Oksanen, T.; Pentti, J.; et al. Antidepressant use before and after the diagnosis of type 2 diabetes: A longitudinal modeling study. Diabetes Care 2010, 33, 1471-1476. [CrossRef] [PubMed]

36. Cleal, B.; Panton, U.H.; Willaing, I.; Holt, R.I. Diabetes and depression in Denmark 1996-2010: National data stratified by occupational status and annual income. Diabet. Med. 2017, 34, 108-114. [CrossRef] [PubMed]

37. Knol, M.J.; Geerlings, M.I.; Grobbee, D.E.; Egberts, A.C.; Heerdink, E.R. Antidepressant use before and after initiation of diabetes mellitus treatment. Diabetologia 2009, 52, 425. [CrossRef] [PubMed]

38. Chen, P.C.; Chan, Y.T.; Chen, H.F.; Ko, M.C.; Li, C.Y. Population-based cohort analyses of the bidirectional relationship between type 2 diabetes and depression. Diabetes Care 2013, 36, 376-382. [CrossRef] [PubMed]

39. Huang, C.J.; Wang, S.Y.; Lee, M.H.; Chiu, H.C. Prevalence and incidence of mental illness in diabetes: A national population-based cohort study. Diabetes Res. Clin. Pract. 2011, 93, 106-114. [CrossRef] [PubMed] 
40. Hsu, Y.M.; Su, L.T.; Chang, H.M.; Sung, F.C.; Lyu, S.Y.; Chen, P.C. Diabetes mellitus and risk of subsequent depression: A longitudinal study. Int. J. Nurs. Stud. 2012, 49, 437-444. [CrossRef] [PubMed]

41. Aarts, S.; Van den Akker, M.; van Boxtel, M.P.; Jolles, J.; Winkens, B.; Metsemakers, J.F. Diabetes mellitus type II as a risk factor for depression: A lower than expected risk in a general practice setting. Eur. J. Epidemiol. 2009, 24, 641-648. [CrossRef] [PubMed]

42. Golden, S.H.; Lazo, M.; Carnethon, M.; Bertoni, A.G.; Schreiner, P.J.; Roux, A.V.; Lee, H.B.; Lyketsos, C. Examining a bidirectional association between depressive symptoms and diabetes. Jama 2008, 299, 2751-2759. [CrossRef] [PubMed]

43. Icks, A.; Albers, B.; Haastert, B.; Pechlivanis, S.; Pundt, N.; Slomiany, U.; Erbel, R.; Jöckel, K.H.; Kruse, J.; Kulzer, B.; et al. Risk for high depressive symptoms in diagnosed and previously undetected diabetes: 5-year follow-up results of the Heinz Nixdorf Recall study. PLoS ONE. 2013, 8, e56300. [CrossRef] [PubMed]

44. Demakakos, P.; Zaninotto, P.; Nouwen, A. Is the association between depressive symptoms and glucose metabolism bidirectional? Evidence from the English Longitudinal Study of Ageing (ELSA). Psychosom. Med. 2014, 76, 555. [CrossRef] [PubMed]

45. Hamer, M.; Batty, G.D.; Kivimaki, M. Haemoglobin A1c, fasting glucose and future risk of elevated depressive symptoms over 2 years of follow-up in the English Longitudinal Study of Ageing. Psychol. Med. 2011, 41, 1889-1896. [CrossRef] [PubMed]

46. Ryu, E.; Chamberlain, A.M.; Pendegraft, R.S.; Petterson, T.M.; Bobo, W.V.; Pathak, J. Quantifying the impact of chronic conditions on a diagnosis of major depressive disorder in adults: A cohort study using linked electronic medical records. BMC Psychiatry 2016, 16, 114. [CrossRef] [PubMed]

47. James, B.O.; Omoaregba, J.O.; Eze, G.; Morakinyo, O. Depression among patients with diabetes mellitus in a Nigerian teaching hospital. S. Afr. J. Psychiatry 2010, 16, 61-64. [CrossRef]

48. Lin, E.H.; Von Korff, M. Mental disorders among persons with diabetes-results from the World Mental Health Surveys. J. Psychosom. Res. 2008, 65, 571-580. [CrossRef] [PubMed]

49. Meurs, M.; Roest, A.M.; Wolffenbuttel, B.H.; Stolk, R.P.; de Jonge, P.; Rosmalen, J.G. Association of depressive and anxiety disorders with diagnosed versus undiagnosed diabetes: An epidemiological study of 90,686 participants. Psychosom. Med. 2016, 78, 233-241. [CrossRef] [PubMed]

50. Clarke, T.K.; Obsteter, J.; Hall, L.S.; Hayward, C.; Thomson, P.A.; Smith, B.H.; Padmanabhan, S.; Hocking, L.J.; Deary, I.J.; Porteous, D.J.; et al. Investigating shared aetiology between type 2 diabetes and major depressive disorder in a population based cohort. Am. J. Med. Genet. Part B Neuropsychiatr. Genet. 2017, 174, 227-234. [CrossRef] [PubMed]

51. Bessel, M.; Vigo, Á.; Poyastro, A.; Nunes, M.A.; Duncan, B.B.; Schmidt, M.I. Stages of hyperglycemia and common mental disorders in adults-The Brazilian Study of Adult Health (ELSA-Brasil). Sao Paulo Med. J. 2016, 134, 423-429. [CrossRef] [PubMed]

52. Yamauchi, T.; Inagaki, M.; Yonemoto, N.; Iwasaki, M.; Akechi, T.; Sawada, N.; Iso, H.; Noda, M.; Tsugane, S. History of diabetes and risk of suicide and accidental death in Japan: The Japan Public Health Centre-based Prospective Study, 1990-2012. Diabetes Metab. 2016, 42, 184-191. [CrossRef] [PubMed]

53. Berge, L.I.; Riise, T.; Fasmer, O.B.; Lund, A.; Oedegaard, K.J.; Hundal, Ø. Risk of depression in diabetes is highest for young persons using oral anti-diabetic agents. Diabet. Med. 2012, 29, 509-514. [CrossRef] [PubMed]

54. van Dooren, F.E.; Denollet, J.; Verhey, F.R.; Stehouwer, C.D.; Sep, S.J.; Henry, R.M.; Kremers, S.P.; Dagnelie, P.C.; Schaper, N.C.; van der Kallen, C.J.; et al. Psychological and personality factors in type 2 diabetes mellitus, presenting the rationale and exploratory results from The Maastricht Study, a population-based cohort study. BMC Psychiatry 2016, 16, 17. [CrossRef] [PubMed]

55. Icks, A.; Kruse, J.; Dragano, N.; Broecker-Preuss, M.; Slomiany, U.; Mann, K.; Jöckel, K.H.; Erbel, R.; Giani, G.; Moebus, S. Are symptoms of depression more common in diabetes? Results from the Heinz Nixdorf Recall study. Diabet. Med. 2008, 25, 1330-1336. [PubMed]

56. Bruce, D.G.; Davis, W.A.; Hunter, M.L.; Peters, K.E.; Davis, T.M.; Starkstein, S.E. Lifetime depression history and depression risk in type 2 diabetes: A. case-control study. J. Diabetes Complicat. 2016, 30, 38-42. [CrossRef] [PubMed]

57. Foran, E.; Hannigan, A.; Glynn, L. Prevalence of depression in patients with type 2 diabetes mellitus in Irish primary care and the impact of depression on the control of diabetes. Ir. J. Med. Sci. 2015, 184, 319-322. [CrossRef] [PubMed] 
58. Albertorio-Diaz, J.R.; Eberhardt, M.S.; Oquendo, M.; Mesa-Frias, M.; He, Y.; Jonas, B.; Kang, K. Depressive states among adults with diabetes: Findings from the National Health and Nutrition Examination Survey, 2007-2012. Diabetes Res. Clin. Pract. 2017, 127, 80-88. [CrossRef] [PubMed]

59. Mäntyselkä, P.; Korniloff, K.; Saaristo, T.; Koponen, H.; Eriksson, J.; Puolijoki, H.; Timonen, M.; Sundvall, J.; Kautiainen, H.; Vanhala, M. Association of depressive symptoms with impaired glucose regulation, screen-detected, and previously known type 2 diabetes: Findings from the Finnish D2D Survey. Diabetes Care 2011, 34, 71-76. [CrossRef] [PubMed]

60. Bouwman, V.; Adriaanse, M.C.; Van't Riet, E.; Snoek, F.J.; Dekker, J.M.; Nijpels, G. Depression, anxiety and glucose metabolism in the general Dutch population: The new Hoorn study. PLoS ONE 2010, 5, e9971. [CrossRef] [PubMed]

61. Li, Z.; Guo, X.; Jiang, H.; Sun, G.; Sun, Y.; Abraham, M.R. Diagnosed but Not Undiagnosed Diabetes Is Associated with Depression in Rural Areas. Int. J. Environ. Res. Public Health 2016, 13, 1136. [CrossRef] [PubMed]

62. Saglam, Z.A.; Saler, T.; Erdem, T.Y.; Ataoglu, E.; Temiz, L.U.; Yenigun, M. The frequency of depression in Turkish patients with diabetes and diabetic complications. Endocrinologist 2010, 20, 19-22. [CrossRef]

63. Kim, W.K.; Shin, D.; Song, W.O. Depression and its comorbid conditions more serious in women than in men in the United States. J. Women's Health 2015, 24, 978-985. [CrossRef] [PubMed]

64. Islam, S.M.; Ferrari, U.; Seissler, J.; Niessen, L.; Lechner, A. Association between depression and diabetes amongst adults in Bangladesh: A hospital based case-control study. J. Glob. Health 2015, 5, 1-9. [CrossRef] [PubMed]

65. Wiltink, J.; Michal, M.; Wild, P.S.; Schneider, A.; König, J.; Blettner, M.; Münzel, T.; Schulz, A.; Weber, M.; Fottner, C.; et al. Associations between depression and diabetes in the community: Do symptom dimensions matter? Results from the Gutenberg Health Study. PLoS ONE 2014, 9, e105499. [CrossRef] [PubMed]

66. Adriaanse, M.C.; Dekker, J.M.; Heine, R.J.; Snoek, F.J.; Beekman, A.J.; Stehouwer, C.D.; Bouter, L.M.; Nijpels, G.; Pouwer, F. Symptoms of depression in people with impaired glucose metabolism or Type 2 diabetes mellitus: The Hoorn Study. Diabet. Med. 2008, 25, 843-849. [CrossRef] [PubMed]

67. Westra, S.; Simsek, S.; Rutters, F.; Krul-Poel, Y.M.; Stehouwer, C.D.; Dekker, J.M.; Pouwer, F. Low vitamin $\mathrm{D}$ levels are not a contributing factor to higher prevalence of depressive symptoms in people with Type 2 diabetes mellitus: The Hoorn study. Diabet. Med. 2017, 34, 577-581. [CrossRef] [PubMed]

68. Singhal, A.; Ross, J.; Seminog, O.; Hawton, K.; Goldacre, M.J. Risk of self-harm and suicide in people with specific psychiatric and physical disorders: Comparisons between disorders using English national record linkage. J. R. Soc. Med. 2014, 107, 194-204. [CrossRef] [PubMed]

69. Webb, R.T.; Kontopantelis, E.; Doran, T.; Qin, P.; Creed, F.; Kapur, N. Risk of self-harm in physically ill patients in UK primary care. J. Psychosom. Res. 2012, 73, 92-97. [CrossRef] [PubMed]

70. Myers, A.K.; Grannemann, B.D.; Lingvay, I.; Trivedi, M.H. Brief report: Depression and history of suicide attempts in adults with new-onset Type 2 Diabetes. Psychoneuroendocrinology 2013, 38, 2810-2814. [CrossRef] [PubMed]

71. Igwe, M.N.; Uwakwe, R.; Ahanotu, C.A.; Onyeama, G.M.; Bakare, M.O.; Ndukuba, A.C. Factors associated with depression and suicide among patients with diabetes mellitus and essential hypertension in a Nigerian teaching hospital. Afr. Health Sci. 2013, 13, 68-77. [CrossRef] [PubMed]

72. Handley, T.E.; Ventura, A.D.; Browne, J.L.; Rich, J.; Attia, J.R.; Reddy, P.; Pouwer, F.; Speight, J. Suicidal ideation reported by adults with type 1 or type 2 diabetes: Results from Diabetes MILES-Australia. Diabet. Med. 2016, 33, 1582-1589. [CrossRef] [PubMed]

73. Sendela, J.; Zdunczyk, B.; Trippenbach-Dulska, H.; Szypowska, A. Prevalence of depressive symptoms in school aged children with type 1 diabetes-A questionnaire study. Psychiatr. Pol. 2015, 49, 1005-1016. [CrossRef] [PubMed]

74. Fuller-Thomson, E.; Sawyer, J.L. Lifetime prevalence of suicidal ideation in a representative sample of Canadians with type 1 diabetes. Diabet. Res. Clin. Pract. 2009, 83, e9-e11. [CrossRef] [PubMed]

75. Batty, G.D.; Kivimaki, M.; Park, I.S.; Jee, S.H. Diabetes and raised blood glucose as risk factors for future suicide: Cohort study of 1234927 Korean men and women. J. Epidemiol. Community Health 2012, 66, 650-652. [CrossRef] [PubMed]

76. Webb, R.T.; Lichtenstein, P.; Dahlin, M.; Kapur, N.; Ludvigsson, J.F.; Runeson, B. Unnatural deaths in a national cohort of people diagnosed with diabetes. Diabetes Care 2014, 37, 2276-2283. [CrossRef] [PubMed] 
77. Davis, W.A.; Starkstein, S.E.; Bruce, D.G.; Davis, T.M. Risk of suicide in Australian adults with diabetes: The Fremantle Diabetes Study. Int. Med. J. 2015, 45, 976-980. [CrossRef] [PubMed]

78. Hasan, S.S.; Mamun, A.A.; Clavarino, A.M.; Kairuz, T. Incidence and risk of depression associated with diabetes in adults: Evidence from longitudinal studies. Community Ment. Health J. 2015, 51, 204-210. [CrossRef] [PubMed]

79. Korczak, D.J.; Pereira, S.; Koulajian, K.; Matejcek, A.; Giacca, A. Type 1 diabetes mellitus and major depressive disorder: Evidence for a biological link. Diabetologia 2011, 54, 2483. [CrossRef] [PubMed]

80. Raison, C.L.; Capuron, L.; Miller, A.H. Cytokines sing the blues: Inflammation and the pathogenesis of depression. Trends Immunol. 2006, 27, 24-31. [CrossRef] [PubMed]

81. Van Harten, B.; de Leeuw, F.E.; Weinstein, H.C.; Scheltens, P.; Biessels, G.J. Brain imaging in patients with diabetes: A systematic review. Diabetes Care 2006, 29, 2539-2548. [CrossRef] [PubMed]

82. Berge, L.I.; Riise, T.; Tell, G.S.; Iversen, M.M.; Østbye, T.; Lund, A.; Knudsen, A.K. Depression in persons with diabetes by age and antidiabetic treatment: A cross-sectional analysis with data from the Hordaland Health Study. PLoS ONE 2015, 10, e0127161. [CrossRef] [PubMed]

83. Oliveira, R.A.; Tostes, M.; Queiroz, V.A.; Rodacki, M.; Zajdenverg, L. Insulin mediated improvement in glycemic control in elderly with type 2 diabetes mellitus can improve depressive symptoms and does not seem to impair health-related quality of life. Diabet. Metab. Syndr. 2015, 7, 55. [CrossRef] [PubMed]

84. Renn, B.N.; Feliciano, L.; Segal, D.L. The bidirectional relationship of depression and diabetes: A systematic review. Clin. Psychol. Rev. 2011, 31, 1239-1246. [CrossRef] [PubMed]

85. Kammer, J.R.; Hosler, A.S.; Leckman-Westin, E.; DiRienzo, G.; Osborn, C.Y. The association between antidepressant use and glycemic control in the Southern Community Cohort Study (SCCS). J. Diabetes Complicat. 2016, 30, 242-247. [CrossRef] [PubMed]

86. Roshanaei-Moghaddam, B.; Katon, W.J.; Russo, J. The longitudinal effects of depression on physical activity. Gen. Hosp. Psychiatry 2009, 31, 306-315. [CrossRef] [PubMed]

87. Nock, M.K.; Borges, G.; Bromet, E.J.; Alonso, J.; Angermeyer, M.; Beautrais, A.; Bruffaerts, R.; Chiu, W.T.; De Girolamo, G.; Gluzman, S.; et al. Cross-national prevalence and risk factors for suicidal ideation, plans and attempts. Br. J. Psychiatry 2008, 192, 98-105. [CrossRef] [PubMed]

88. Yoshimasu, K.; Kiyohara, C.; Miyashita, K. Suicidal risk factors and completed suicide: Meta-analyses based on psychological autopsy studies. Environ. Health Prev. Med. 2008, 13, 243. [CrossRef] [PubMed]

89. Mars, B.; Burrows, S.; Hjelmeland, H.; Gunnell, D. Suicidal behaviour across the African continent: A review of the literature. BMC Public Health 2014, 14, 606. [CrossRef] [PubMed]

90. World Health Organization. Preventing Suicide: A Global Imperative; WHO: Geneva, Switzerland, 2014.

91. Goldsmith, S.K. Reducing Suicide: A National Imperative; National Academy Press: Washington, DC, USA, 2002.

92. Daigle, M. MMPI inmate profiles: Suicide completers, suicide attempters, and non-suicidal controls. Behav. Sci. Law 2004, 22, 833-842. [CrossRef] [PubMed]

93. DeJong, T.M.; Overholser, J.C.; Stockmeier, C.A. Apples to oranges?: A direct comparison between suicide attempters and suicide completers. J. Affect. Disord. 2010, 124, 90-97. [CrossRef] [PubMed]

(C) 2018 by the authors. Licensee MDPI, Basel, Switzerland. This article is an open access article distributed under the terms and conditions of the Creative Commons Attribution (CC BY) license (http://creativecommons.org/licenses/by/4.0/). 